\title{
Crise, essai, discipline : Autour de Robert Musil
}

\author{
Par THOMAS BOLMAIN \& ANDREA CAVAZZINI
}

Université de Liège

\section{Liminaire}

Au croisement de la philosophie, de la littérature et de la pensée politique, cet article se propose d'interroger le travail d'essayiste et de romancier de Robert Musil (1880-1942) par l'investigation de trois notions : la crise, l'essai, la discipline.

On sait que cette entreprise romanesque - qui culmine dans la rédaction d'un livre demeuré inachevé, L'Homme sans qualités $(1930,1932)$ - compose l'une des œuvres les plus caractéristiques et les plus brillantes du modernisme littéraire européen ; on sait d'autre part que l'immense érudition qui s'y fait jour a sa source dans une formation variée, d'ordre notamment philosophique: proche d'Alexius Meinong ${ }^{1}$, Musil avait réalisé, sous la direction de Carl Stumpf, une thèse consacrée au positivisme d'Ernst Mach. Ce point restera ici inentamé. Le propos trouvera plutôt le lieu de sa cohérence et de son unité dans un certain espace-temps historique : centré sur la période critique s'étalant de l'avant-guerre 14-18 (moment où se déroule l'action de L'Homme sans qualités) à l'entre-deux guerres (moment où l'ouvrage est effectivement écrit), il a pour centre de gravité géographique l'Europe centrale, en particulier Vienne. Aussi tentera-t-on dans ces pages non seulement d'étudier, mais encore de mettre en contexte l'œuvre de Musil, de rendre compte de ce que l'on pourrait appeler l'environnement « spirituel » où s'inscrit sa pensée, en multipliant les coups de sonde chez des

1 Cf. Allen Thiher, Understanding Robert Musil, Columbia (South Carolina), University of South Carolina Press, 2009, p. 5. 
auteurs contemporains (Husserl, en philosophie ; Lukács, entre philosophie et politique) ou même plus tardifs (Thomas Bernhard).

\section{Contexte. Sept vignettes introductives}

1. - Soit une définition formelle du concept de crise. Appelons crise le moment qui introduit dans l'histoire - sociale, politique, économique, épistémique, culturelle - une différence, la différence séparant un " déjà plus » d'un « pas encore ». La crise, comme « rupture » et traçage d'un seuil, « vise [alors] la pointe d'intensité dans l'histoire, [...] la coupure entre deux périodes radicalement différentes de cette histoire [...], l'échéance d'un long processus qui vient à éclater ${ }^{1}$. Moment de transition, elle constitue pour la pensée qui se propose de réfléchir cette histoire sous l'une ou l'autre forme (philosophique ou littéraire, politique) un instant d'incertitude, voire de profonde inquiétude: inconfortablement logée le cul entre deux chaises, la pensée est alors tiraillée, partagée entre l'impression d'un proche effondrement et celle d'un recommencement tout éventuel. La perte des repères, des critères qui, habituellement, orientaient son jugement, qui le déterminaient, lui impose désormais de s'essayer à réfléchir les signes ou les symptômes à partir desquels décider du sens possible de ce présent détraqué ; elle l'oblige, en somme, à tenter une opération de diagnostic. Nous trouvons dans un roman autrichien de 1963 la formule littéraire d'un tel sentiment de la crise :

Quelque chose de neuf se tenait en attente derrière tout cela, mais je ne pouvais pas le voir car ma tête était remplie de vieilles images et mes yeux incapables de changer leur façon de voir. J'avais perdu l'ancien mais je n'avais pas encore gagné ce qui était nouveau; ce nouveau me restait inaccessible mais je savais qu'il existait ${ }^{2}$.

${ }^{1}$ Michel Foucault, «La politique est la continuation de la guerre par d'autres moyens », dans Id., Dits et écrits, t. 1, Paris, Éditions Gallimard, coll. "Quarto », 2001, p. 1571.

${ }_{2}^{2}$ Marlen Haushofer, Le Mur, trad. fr. J. Chambon, Arles, Éditions Actes Sud, coll. «Babel», 2009, p. 156 (nous soulignons). On pensera aussi à la préface de Hannah Arendt, La Crise de la culture. Sa portée sociale et politique, trad. fr. P. Lévy et. al., Paris, Éditions Gallimard, coll. « Folio », 1972, intitulée « La brèche entre le passé et le futur» (trad. fr. J. Bontemps et P. Lévy). S'appuyant notamment sur Kafka, Arendt y décrit cet « intervalle dans le temps qui est entièrement déterminé par des choses qui ne sont plus et par des choses qui ne sont pas encore »; or ce moment où 
2. - «Crise » est devenu le signifiant maître d'une époque - l'époque présente - qui s'est dramatiquement révélée n'être pas à la hauteur des récits émancipateurs qui structurèrent l'imaginaire de la modernité : crises économiques, crise écologique, crise de la représentation parlementaire qui ne représente décidément plus qu'elle-même - , crise, enfin, de la fonction-intellectuelle critique, notamment dans le domaine de l'art. À l'autre extrémité de cette période, la modernité philosophique elle-même s'inaugurait par le constat d'une crise, entériné par la critique kantienne ; une crise qui trouvait son répondant historico-politique dans la Révolution française et dans l'accession de la classe bourgeoise à une position dominante mais contrariée dans le champ de la culture.

Dans L'Absolu littéraire (1978), Philippe Lacoue-Labarthe et Jean-Luc Nancy rapportent la possibilité de la naissance de la littérature moderne - la littérature lorsqu'elle cesse d'être confondue avec un secteur des BellesLettres - à l'existence de cette crise historico-philosophique : autour des frères Schlegel, le "premier romantisme », ou " romantisme d'Iéna », présente « la formulation la plus proprement critique [...] de la crise de l'histoire moderne $»^{1}$. Or, en constituant la littérature en tant qu'absolu autrement dit: en tant qu'elle s'auto-produit absolument par le biais de la création, immanente à son existence, de sa propre théorie critique -, le «romantisme théorique» expérimenté dans la revue de L'Athenaeum définirait en fait par anticipation les schèmes avant-gardistes qui vont circonscrire l'espace du développement de tout le modernisme littéraire ${ }^{2}$. Aussi bien,

se rompt le fil de la tradition représente pour l'exercice de la pensée non seulement le risque mais encore la chance suprêmes, en fait «le moment de la vérité » (p. 19), puisqu'il découvre alors l'occasion de se faire «politique», par le biais d'une nécessaire pratique de l'« essai » et de l'« expérience» (p. 25) : «Ce n'est que dans la mesure où il pense $[\ldots]$ que l'homme dans la pleine réalité de son être concret vit dans cette brèche du temps entre le passé et le futur. Cette brèche $[\ldots]$ il se peut bien qu'elle soit la région de l'esprit ou, plutôt, le chemin frayé par la pensée, ce petit tracé de non-temps que l'activité de la pensée inscrit à l'intérieur de l'espace-temps des mortels et dans lequel le cours des pensées, du souvenir et de l'attente sauve tout ce qu'il touche de la ruine du temps historique et biographique. [...] Mais l'ennuyeux est que nous ne semblons ni équipés ni préparés pour cette activité de pensée d'installation dans la brèche entre le passé et le futur » (p. 24).

${ }^{1}$ Philippe Lacoue-Labarthe et Jean-Luc Nancy, L'Absolu littéraire. Théorie de la littérature du romantisme allemand, Paris, Éditions du Seuil, coll. "Poétique», 1978, p. 13-14.

${ }^{2}$ Ibid., p. 21-22, 26. 
si le romantisme est abordable [...], ce ne peut être d'une certaine façon que dans «l'entre-deux», [...] comme irruption, événement, surgissement ou surrection $[\ldots]$, bref tout ce qu'on a raison de référer à quelque chose comme une crise $e^{1}$.

Mieux, s'il a pu contribuer à définir « un véritable inconscient» à l'œuvre « dans la plupart des grands motifs de notre "modernité" », c'est parce qu'au fond tout se passait alors comme si les différents auteurs concernés avaient à leur tour

eu conscience [...] que le monde (et pas seulement les Lettres) était en train de changer d'époque ou de tourner sur lui-même, ouvrant sans doute une perspective illimitée, mais n'offrant rien dans l'immédiat qui fût à la mesure de l'événement ${ }^{2}$.

Clôturant philosophiquement et historiquement ce moment critique, situé, donc, à une distance permettant de le juger, Hegel décrira, dans la Préface de la Phénoménologie de l'Esprit (1807), «l'ébranlement [...] [d'un] monde » révélé par de vagues indices, "l'insouciance et l'ennui qui envahissent ce qui subsiste encore, le pressentiment vague d'un inconnu », autant de «signes précurseurs de quelque chose d'autre qui se prépare $»^{3}$. Plus près de la période considérée aujourd'hui, Nietzsche, dans Le Gai savoir $(1882,1887)$, montrera pour sa part que les médecins de la civilisation, les critiques de la culture, sont "placés entre aujourd'hui et demain et écartelés dans la contradiction entre aujourd'hui et demain », que leur lieu de prédilection est toujours une « époque de transition fragile et brisée » ${ }^{4}$.

Entre le moment actuel et le lieu, disons kantien et romantique, de sa fondation, cet article souhaite interroger la formulation littéraire d'une « époque de transition » donnée — Vienne, l'entre-deux guerres —, animé par la conviction qu'une telle expérience littéraire articule un certain nombre de motifs qui restent opérants pour penser le temps présent; nul besoin d'y

${ }^{1}$ Ibid., p. 42.

${ }^{2}$ Ibid., p. 26, 19

${ }^{3}$ Georg Wilhelm Friedrich Hegel, Préface à la Phénoménologie de l'Esprit, trad. fr. J. Hyppolite, Paris, Éditions Aubier-Montaigne, coll. «Bibliothèque Philosophique Bilingue », 1966, p. 32-33. Voir Jürgen Habermas, Le Discours philosophique de la modernité. Douze conférences, trad. fr. C. Bouchindhomme et R. Rochlitz, Paris, Éditions Gallimard, coll. « NRF », 1986, p. 7.

4 Friedrich Nietzsche, Le Gai savoir, trad. fr. P. Wotling, Paris, Éditions Flammarion, coll. « GF Flammarion », 2007, p. 284 (§ 383), 343 (§ 377). 
insister, la crise, l'essai et la discipline forment bien un ensemble de notions qui est tout sauf étranger à notre actualité. Mais le registre littéraire doit avant tout — puis devra constamment — être rapporté à ses implications philosophiques et politiques.

3. - À suivre en effet Lacoue-Labarthe et Nancy, « il n'y a de crise, en son fond, que philosophique $\gg{ }^{1}$. La plus haute formulation, en philosophie, de la période critique envisagée ici même se trouve chez Husserl, dans la Krisis (1935-1936). Sous la funeste pression conjuguée du naturalisme (de l'objectivisme) et du scepticisme, la rationalité philosophique moderne, définie par la visée de l'universalité et l'infinité de ses tâches, est entrée en crise. Cette crise philosophique est indissociable d'une crise de la raison scientifique; plus précisément, le dévoiement de l'idéal théorétique, fondement constitutif de l'humanité européenne et de son esprit spécifique, sa déviation dans la forme unilatérale d'un idéal d'abstraction et de calculabilité intégrales, a voilé le lieu même de naissance du questionnement philosophico-scientifique, l'étonnement de l'homme devant le mondeambiant, devant le monde-de-la-vie (Lebenswelt). Cette crise philosophique se prolonge en une crise éthique, tant elle concerne le sens et l'existence de l'humanité en général :

La crise de la philosophie a la signification d'une crise de toutes les sciences modernes en tant que membres de l'universalité philosophique : c'est, d'abord de façon latente, puis de plus en plus manifestement, une crise de l'humanité européenne elle-même quant à ce qui donne globalement sens à sa vie culturelle, à l'ensemble de son « existence $»^{2}$.

Si cette situation critique impose aux «philosophes d'aujourd'hui» une pénible « contradiction existentielle ${ }^{3}$, elle n'en ouvre pas moins, conformément à l'analyse précédente, la possibilité d'un diagnostic: la prise en compte des «innombrables symptômes de la décadence de la vie » ${ }^{4}$ permettra même la prescription d'un remède. Il n'en va pas autrement chez Husserl que chez Kant : la crise de la raison n'implique pas de piteusement

\footnotetext{
${ }^{1}$ Philippe Lacoue-Labarthe et Jean-Luc Nancy, L'Absolu littéraire, op. cit., p. 42.

2 Edmund Husserl, La Crise des sciences européennes et la phénoménologie transcendantale, trad. fr. G. Granel, Paris, Éditions Gallimard, coll. « Tel», 2004, $\S 5$, p. 18.

${ }^{3}$ Ibid., $\$ 6$, p. 23.

${ }^{4}$ Edmund Husserl, « La Crise de l'humanité européenne et la philosophie », dans Id., La Crise des sciences européennes et la phénoménologie transcendantale, op. cit., p. 382 .
} 
recourir à une quelconque forme d'irrationalisme; elle impose plutôt une refondation de la rationalité par elle-même, geste dont la condition, ici, est la conversion phénoménologique. La crise philosophico-scientifique, qui est en dernière analyse d'ordre existentielle, force ainsi à la décision éthique, elle contraint à un changement radical d'attitude, à « une révolution volontaire inconditionnée ${ }^{1}$ de l'homme opérée dans la forme d' ' un combat pour se rendre lui-même vrai $»^{2}$. C'est à ce prix que l'on tranchera correctement, de façon à éviter le « brasier nihiliste », l'alternative de l' « héroïsme » et de « la barbarie » qui clôt la conférence « La Crise de l'humanité européenne et la philosophie » prononcée par Husserl, à Vienne, en 1935. C'est enfin dans cette perspective, qui redécouvre dans sa radicalité originaire l'attitude théorétique masquée par les Temps Modernes cartésiens, que pourra s'effectuer « une synthèse de l'universalité théorétique et de la praxis dont l'intérêt est universel », vers " une critique de l'humanité elle-même», une autocritique méditative productrice $d$ '« une humanité fondamentalement nouvelle $»^{3}$.

Relisant cette dernière conférence, Milan Kundera notera que le roman européen, depuis Don Quichotte, « dessine comme une histoire parallèle des Temps Modernes ». À suivre l'auteur de L'Insoutenable légèreté de l'être, rien n'empêche en effet d'écrire une autre histoire de la modernité, injustement négligée par Husserl, une histoire où "l'être de l'homme », certes « oublié » par les rationalités philosophique et scientifique, s'est en revanche vu constitué comme objet central d'une " exploration » expérimentale indéfinie : l'histoire de «l'esprit du roman $»^{4}$. Musil — cela transparaît particulièrement dans ses essais, dans l'essai comme forme autonome, qu'il affectionnait - se situe au croisement de ces deux histoires de l'esprit européen, philosophique ou romanesque. Mais, dans son cas, la crise « épochale », outre ses déterminations philosophico-scientifiques et éthicoexistentielles, apparaît également dans sa dimension politique - ce qui impose un détour.

${ }^{1}$ Ibid., p. 361.

2 Edmund Husserl, La Crise des sciences européennes et la phénoménologie transcendantale, op. cit., §5, p. 18.

${ }^{3}$ Edmund Husserl, « La Crise de l'humanité européenne et la philosophie », dans Id., La Crise des sciences européennes et la phénoménologie transcendantale, op. cit., p. 382-383, 363.

${ }^{4}$ Milan Kundera, L'Art du roman, Paris, Éditions Gallimard, coll. « Folio», 2003, p. 14-15, 30. Voir Thomas Bolmain, «Foucault lecteur de Husserl. Articuler une rencontre », dans Bulletin d'analyse phénoménologique, vol. 4, n 3, 2008, p. 215 sq.

95 
4. - Au début des années 1920, Lukács rédige à Vienne les essais composant le recueil Histoire et conscience de classe (1923), ouvrage fondateur du «marxisme occidental ${ }^{1}$. Partageant avec son contemporain Karl Korsch le constat d'une «crise décisive du marxisme» liée à une occultation de la dimension proprement philosophique de son origine ${ }^{2}$, Lukács s'emploie à refonder le matérialisme historique par le biais d'un retour critique à la philosophie hégélienne et aux catégories de négation, de totalité et de conscience de soi. La possibilité même d'une telle entreprise, Lukács la découvre dans la crise actuelle de la pensée bourgeoise, une crise d'abord économique, mais qui s'éprouve aussi dans l'ordre de la culture ou de l'idéologie ${ }^{3}$. La conséquence essentielle du devenir-monde de l'économie, qu'on mettra à l'actif du capitalisme industriel, est le morcellement, la fragmentation de la vie sociale en une multiplicité de sphères ou de champs hétérogènes, séparés, entre lesquels ne subsiste d'autre solution de continuité qu'abstraite. Inscrite dans l'ordre politique des choses par l'apparition de la bureaucratie, et dans l'ordre scientifique du monde par une rationalisation calculante synonyme de «désenchantement » (l'Entzauberung wébérienne), cet état de fait repose en ultime instance sur une recomposition fondamentale de l'espace-temps social conditionnée par la marchandisation du travail, puis par sa division tayloriste. Celles-ci ont de graves conséquences existentielles, que résume le concept de réification (Verdinglichung), en une extrapolation magistrale de l'analyse - entreprise par Marx au Livre I du Capital — du « fétichisme de la marchandise » :

L'universalité de la forme marchande conditionne [...], tant sur le plan subjectif que sur le plan objectif, une abstraction du travail humain qui s'objective dans les marchandises [...]. Le temps de travail socialement nécessaire, fondement du calcul rationnel, est produit [...], grâce à une mécanisation et à une rationalisation toujours plus poussée du processus de travail, comme une quantité de travail objectivement calculable qui s'oppose au travailleur en une objectivité achevée et close. Avec la décomposition moderne «psychologique » du processus de travail (système de Taylor), cette mécanisation rationnelle pénètre jusqu'à l'«âme » du travailleur : même ses

\footnotetext{
${ }^{1}$ Maurice Merleau-Ponty, Les Aventures de la dialectique, Paris, Éditions Gallimard, 1955, p. 43-80 ; Perry Anderson, Sur le marxisme occidental, trad. fr. D. Letellier et S. Niémetz, Paris, Éditions Maspero, 1977.

${ }^{2}$ Karl Korsch, Marxisme et philosophie, trad. fr. B. Dericquebour et alii, Paris, Éditions Allia, 2009, p. 59.

${ }^{3}$ Georg Lukács, Histoire et conscience de classe, trad. fr. K. Axelos et J. Bois, Paris, Éditions de Minuit, coll. « Arguments », 2010, p. 92.
} 
propriétés psychologiques sont séparées de l'ensemble de sa personnalité et sont objectivées ${ }^{1}$.

Ainsi remonte-t-on au fondement historique, épistémique, économique et politique, de la réification, à la séparation du producteur et de son produit celui-ci lui faisant désormais face comme une réalité étrangère et autosuffisante -, laquelle détermine les formes de sa fausse conscience, son apathie et son caractère contemplatif, tout comme elle définit, à un autre niveau, les formes de la fausse conscience typiquement bourgeoise (dans le droit ou la philosophie). C'est dire que « la séparation de la force de travail et de la personnalité de l'ouvrier» a «imprimé sa structure à toute la conscience de l'homme », qu'elle «s'est enfoncée dans l'“éthique" » comme au plus profond du $«$ psychique $»^{2}$ :

Plongés dans ce temps abstrait, exactement mesurable, le temps qui est devenu l'espace de la physique, et qui est en même temps une condition, une conséquence de la production spécialisée et décomposée [...] de l'objet du travail, les sujets doivent eux aussi être nécessairement décomposés rationnellement d'une manière correspondant. D'un côté, $[. .$.$] leur travail parcellaire$ mécanisé $[. .$.$] est transformé en réalité quotidienne durable et insurmontable$ [...]. De l'autre côté, la décomposition mécanique [...] rompt aussi les liens qui, dans la production «organique», reliaient à une communauté chaque sujet du travail, pris un à un ${ }^{3}$.

C'est de cette façon que Lukács relie pour sa part rationalités scientifique, philosophique et questionnement éthico-existentiel, en les rapportant à leur véritable soubassement, qui est d'ordre économique.

Quant au versant positif de son analyse, on se bornera à rappeler que, dans ce contexte, la crise économique dit aussi bien « le problème qui oppose à la pensée économique de la bourgeoisie une barrière infranchissable ». Car si la formalisation, l'abstraction extrême de la "science » économique vaut comme ultime «barrière méthodologique à la compréhension de cette crise », la pensée bourgeoise ne peut alors plus dissimuler qu'elle rencontre, dans cette formalisation de la crise elle-même, " sa limite » : réapparait ainsi, derrière l'atomisation ou la parcellarisation du monde social, la totalité qu'il forme en vérité ${ }^{4}$. Lorsque «les objets de l'expérience sont saisis et compris

\footnotetext{
${ }^{1}$ Ibid., p. 114-115.

2 Ibid., p. 128-129.

${ }^{3}$ Ibid., p. 117-118.

${ }^{4}$ Ibid., p. 135, 130.
} 
comme des moments de la totalité, c'est-à-dire comme des moments de l'ensemble de la société en plein bouleversement historique ${ }^{1}$, la conscience réifiée trouve l'occasion de son reversement dialectique en une authentique conscience de classe. Celle-ci, qui est moins conscience savante de la totalité que conduite pratique et militante visant la transformation de la totalité sociale, jugera, orientera et agira ses intérêts à partir de sa situation, en tant qu'elle est immanente à, et déterminée par et dans, le procès de la production. L'être chosifié apparaît alors comme devenir historique, l'immédiateté de ce qui se donnait comme pur quantitatif découvre, par médiations concrètes et négations déterminées, son essence qualitative, et le prolétariat, d'abord "pur et simple objet du devenir social », se reconnait comme « sujet de sa propre vie $»^{2}$. Reposant in fine sur l'aptitude autocritique $»^{3} \mathrm{du}$ prolétariat comme classe - Merleau-Ponty y insistera largement dans Les Aventures de la dialectique - , la conception de Lukács culmine dans une articulation de la théorie et de la pratique retrouvant les implications extrêmes du concept marxien de praxis, de praktisch-kritischen Tätigkeit (première thèse sur Feuerbach) :

Seule la classe peut, par son action, pénétrer la réalité sociale et la transformer en sa totalité. C'est pourquoi, étant considération de la totalité, la « critique » qui s'exerce à partir de ce point de vue est l'unité dialectique de la théorie et de la praxis ${ }^{4}$.

Alors, comme l'écrira, en 1967, dans une perspective strictement analogue, Guy Debord, «la théorie n'a plus à connaître que ce qu'elle fait » ${ }^{5}$. Mais, il faut le souligner, Lukács insiste encore longuement sur la double condition empirique donnant son efficace concrète à une conscience praxique de classe qui demeure, avant tout, l'expression d'une nécessité historique. Il s'agit d'abord de l'importance du Parti, comme vecteur d'organisation de la conscience prolétarienne : car, à dire vrai, «l'organisation est la forme de la médiation entre la théorie et la pratique $»^{6}$. Pour autant, la transformation

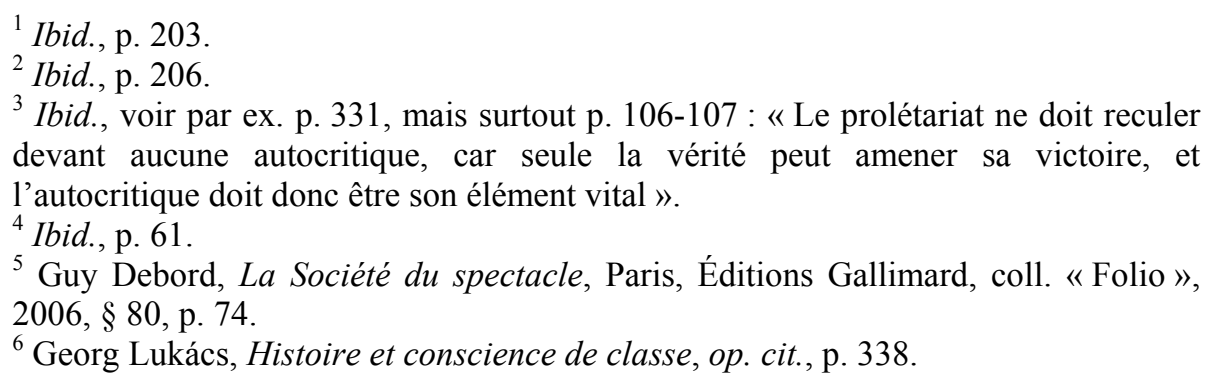

98 
consciente du mode de production capitaliste trouve sa source ultime dans un engagement éthico-existentiel « discipliné » : «Pour conquérir les conditions sociales de la liberté réelle, il faut livrer des batailles au cours desquelles ne disparaitront pas seulement la société actuelle, mais aussi le type humain produit par cette société »; or, réciproquement, circulairement, cela suppose un "engagement actif de l'ensemble de la personnalité», du «cœur de [1'] existence $»{ }^{1}$. Si le devenir-marchandise du monde avait pour corrélat l'effacement de l'expérience vécue en première personne, «l'attitude réellement pratique » ne pourra signifier que la reconquête et la «mise en jeu de toute la personnalité $»^{2}$.

5. - Au début d'un bref essai intitulé La Phrase infinie de Thomas Bernhard, le philosophe italien Aldo G. Gargani relève que « l'originalité de la littérature autrichienne », de Musil à Bernhard, "repose sur la dissolution de l'épistémologie fondationnelle qui place en correspondance le langage et le monde $»^{3}$. Il y va, en somme, d'une crise de la représentation, d'une crise affectant la rationalité qui reliait selon une corrélation univoque le langage et son référent réputé non problématique, le réel. Comme l'auteur le précise ailleurs ${ }^{4}$, un grave soupçon pèse désormais sur la raison - dont les règles, jusque-là, paraissaient strictement déterminantes —, depuis que l'on s'est avisé que ces règles ne sont rien d'autre que le «reflet de pratiques linguistiques qui, comme telles, échappent à toute justification »; depuis que l'on s'est aperçu — particulièrement avec Wittgenstein — que quoique l'usage du langage impose de faire comme si nos propositions « se référaient à une réalité existant par elle-même », il n'en reste pas moins qu'il est impossible de « saisir cette réalité autrement qu'au moyen du langage ». Gargani poursuit en suggérant que cette «dissolution de la rationalité contraignante $[\ldots]$ s'étend $[. .$.$] à la destruction du concept de Sujet issu de la$ tradition propre à la culture occidentale $»$.

Ce moment négatif n'est pourtant pas le dernier mot de l'auteur. Indépendamment de la perspective qui lui est propre - l'approfondissement $\mathrm{du}$ symbolisme linguistique doit libérer, en rapport avec la notion de décision, une autre pensée du temps et de la nouveauté —, Gargani relève

${ }^{1}$ Ibid., p. 356, 355, 359.

${ }^{2}$ Ibid., p. 360. Pour prolonger l'analyse, voir Andrea Cavazzini, Enquête ouvrière et théorie critique, Liège, Presses Universitaires de Liège, coll. « Philosophie », 2013.

${ }^{3}$ Aldo Giorgio Gargani, La Phrase infinie de Thomas Bernhard, trad. fr. J.-P. Cometti, Paris, Éditions de L'Éclat, 1990, p. 7.

${ }^{4}$ Sur ce qui suit, voir Aldo Giorgio Gargani, L'Étonnement et le hasard, trad. fr. J.-P. Cometti et J. Hansen, Paris, Éditions de L'Éclat/Chemin de ronde, 1988, p. 9-10, 13, 15. 
que cette dissolution de la rationalité et du sujet classiques est positivement compensée, «dans la culture autrichienne », par l'exigence, assumée dans le langage, notamment littéraire, d'une "étroite solidarité [...] de l'éthique et de l'esthétique $»^{1}$. C'est un tel mouvement de destruction/recomposition qu'il décrit, par exemple chez Musil. En effet, d'une part,

de diverses façons, Musil, Wittgenstein et Kafka ont observé une diminution de la clarté des significations associées aux circonstances de la vie humaine sous l'effet des automatismes de type causal et des modèles mécaniques [...]; ils ont constaté une diminution de la motivation et de la responsabilité dans la société bureaucratique, assujettie au pouvoir de la répétition dont l'influence s'est même étendue aux styles de l'activité intellectuelle. Tout en augmentant la difficulté qu'il y a à produire un sens, une vérité, une compréhension, et jusqu'à la difficulté de faire une expérience, les aspects de la vie humaine n'ont plus pour seul caractère que celui de l'effet provoqué ${ }^{2}$.

Mais précisément, d'autre part, dans ce contexte où le langage se voit séparé de la référence à la « représentation vécue, à la sensation intérieure $»^{3}$, le lien serré qui, chez Musil, « unit l'éthique et l'esthétique», doit permettre de retrouver, pour la fonder à nouveaux frais, « la possibilité même de vivre une expérience » :

Dans l'œuvre de Musil, et tout particulièrement dans L'Homme sans qualités, la recherche de la motivation est très étroitement associée à l'idée d'une urgence qui vise à nous soustraire à la mécanisation [...], à la concaténation causale et à la légalité mécanique qui marque les événements. La fin en est la découverte d'un ordre de possibilités alternatives où l'humanité, retrouvant un sens menacé par la civilisation contemporaine, pourrait prendre sa place. Aussi Musil, loin de voir dans le langage un outil [...], recherche-t-il en lui une possibilité neuve de s'emparer de l'irréalité [...], de l'ordre pluriel auquel appartiennent les événements ${ }^{4}$.

La suite de cet article entend décrire avec rigueur l'« ordre de possibilités alternatives » dégagé par Musil. Cet ordre se compose pour l'essentiel de deux dimensions : il y va, d'un côté, de la recherche d' " un autre état » (den anderen Zustand), dont Musil propose une ébauche théorique, mais qu'il approchait sans doute déjà, par un autre biais, dans Les Désarrois de l'élève

${ }^{1}$ Aldo Giorgio Gargani, La Phrase infinie de Thomas Bernhard, op. cit., p. 7.

${ }^{2}$ Aldo Giorgio Gargani, L'Étonnement et le hasard, op. cit., p. 101.

${ }^{3}$ Ibid., p. 100.

${ }^{4}$ Aldo Giorgio Gargani, La Phrase infinie de Thomas Bernhard, op. cit., p. 8-9. 
Törless (1906), le roman de jeunesse qui le fit connaître ; on sera attentif, d'un autre côté, à la thématisation de la notion d'essai, non seulement comme genre littéraire à part entière, mais aussi - et peut-être plus profondément - , cette fois non loin de Bernhard, comme motif indispensable à une pensée éthique de la formation disciplinée de soi par soi, d'une réforme décidée du caractère.

Avant d'en venir à ces deux points, et de conclure sur le dernier d'entre eux, nous exposerons la pensée musilienne de la crise historique, de l'époque comme période critique, notamment telle qu'elle apparaît dans L'Homme sans qualités.

6. - Même s'il est vrai, comme le concède Musil, que l'" on ne réfute pas une époque », que l'on ne saute pas par-dessus son propre temps et qu'il faut donc se borner à " lui surveiller les doigts et, de temps en temps, taper dessus ${ }^{1}$, il convient de ne surtout pas « fuir le présent $»^{2}$. C'est le risque et la chance d'une époque critique que de révéler des "symptômes » ${ }^{3}$ autorisant à son endroit un diagnostic :

L'idée que les cultures périssent par épuisement interne est plausible [...]. Que l'on y puisse distinguer des phases d'essor et de déclin corrélatives, également. [...] Les époques tardives sont si hétérogènes, et les cultures, dans ces époques de civilisation, si promptes à s'écrouler comme des montagnes ${ }^{4}$.

En quoi sa propre époque est-elle selon Musil une époque de crise (au sens envisagé plus haut) ? Une "époque tardive » est une époque de civilisation, non de culture ${ }^{5}$. C'est ce qui apparaît lorsqu'elle est mise en contraste avec l'époque qui la devance exactement, c'est-à-dire, dans le cas de Musil, avec

${ }^{1}$ Robert Musil, «Esprit et expérience. Remarques pour des lecteurs réchappés du déclin de l'Occident", dans Id., Essais. Conférences, critiques, aphorismes et réflexions, trad. fr. P. Jaccottet, Paris, Éditions du Seuil, 1984, p. 104.

2 Robert Musil, "L'Allemand comme symptôme », dans Id., Essais. Conférences, critiques, aphorismes et réflexions, op. cit., p. 346.

${ }^{3}$ Voir par ex. Robert Musil, «Esprit et expérience », art. cit., p. 116, et « L’Europe désemparée ou petit voyage du coq à l'âne", dans Id., Essais. Conférences, critiques, aphorismes et réflexions, op. cit., p. 135.

${ }^{4}$ Robert Musil, «Esprit et expérience », art. cit., p. 112-113.

${ }^{5}$ Ibid., p. 114. Voir également Robert Musil, L'Homme sans qualités, t. 1, trad. fr. P. Jaccottet, Paris, Éditions du Seuil, coll. « Points », 1982, p. 121.

101 
« la période qui précède de peu $1900 »{ }^{1}$. Le résultat de l'opération s'exprime de la façon suivante :

Il y a eu à l'origine, dans ce chaos intellectuel, une sorte d'état de polarisation, l'illusion d'une cohérence des buts qui s'est ensuite relâchée pour faire place à une autre, plus faible, au moment de l'expressionisme, et qui se relâche derechef actuellement, pour ne plus nous laisser qu'un «état non orienté », une pagaille découragée ${ }^{2}$.

L'interprétation habituelle d'un tel état de fait s'exprimerait en termes de décadence; or c'est elle que Musil entend rejeter absolument, retrouvant par là le sens authentique de la notion de crise: "L'état actuel de l'esprit européen n'est pas, à mon sens, la décadence, mais une transition encore en cours ; non pas un excès, mais une insuffisante maturité ${ }^{3}$. C'est ce qu'expose encore, dans son registre propre, L'Homme sans qualités, en un chapitre intitulé de façon suggestive «Une mystérieuse maladie d'époque» :

C'est à la fois tout et rien ; on dirait que le sang, ou l'air, ont changé ; une mystérieuse maladie a détruit le germe de génie de l'époque précédente, mais tout reluit de nouveauté, de sorte qu'on ne sait plus en fin de compte, si le monde a réellement empiré, ou si l'on a tout simplement vieilli. Alors, un nouvel âge a décidément commencé ${ }^{4}$.

En somme, si, aux yeux d'Ulrich, le personnage central du livre, «le temps se déplaçait», il n'en ignorait pas moins « où il allait». Pour autant, lorsque l'on a «perdu l'ancien » sans avoir déjà « gagné ce qui était nouveau », tout devient en vérité possible, le meilleur comme le pire. Le pire s'incarne dans « cette fameuse incohérence des idées, cette prolifération privée de centre qui caractérisent le temps présent ${ }^{5}$, dans cette «situation incohérente, disloquée et malheureuse de l'esprit » qui permet par exemple à un journal de qualifier de « génial » un cheval de course, plongeant dès lors Ulrich dans un abîme de perplexité (au $\S 13$ de L'Homme sans qualités). Quant au meilleur que recèlerait éventuellement ce moment critique, il tient en ceci qu'il n'est pas impossible, selon Musil, que ce temps - à la condition de ce qu'il

${ }^{1}$ Robert Musil, «L'Allemand comme symptôme », art. cit., p. 355. Voir également Robert Musil, L'Homme sans qualités, t. 1, op. cit., p. 63-65.

${ }^{2}$ Ibid., p. 355-356.

${ }^{3}$ Ibid., p. 345 (nous soulignons), ou encore p. 362.

${ }^{4}$ Ibid., p. 68.

${ }^{5}$ Ibid., p. 14, 22 
nomme « une politique d'organisation de l'esprit», d'un travail de mise en « ordre » spirituel — finalement « prenne forme » 1 .

7. - À suivre Musil, une telle mise en forme du temps suppose de ne pas demeurer en " arrêt sur le seuil de l'ultime étape à franchir » ${ }^{2}$, mais de prendre la décision d'orienter la vie de l'esprit, et sa propre existence, avec discipline. Or il est possible d'interpréter le personnage d'Ulrich comme cet « ego expérimental ${ }^{3}$ justement chargé d'éprouver, dans l'espace langagier du roman, les implications les plus diverses d'un tel arrêt au lieu du seuil, au lieu de l'entre-deux ou de la transition. Ulrich n'est-il pas cet être de papier qui expérimente in situ une inaptitude radicale à se décider, à trancher dans la crise épochale afin de libérer et de rejoindre d'autres possibilités sociohistoriques?

En tant que sujet, Ulrich est a priori a-morphe (on y reviendra); c'est dire qu'il est essentiellement fonction des formes objectives que lui propose l'époque comme espace de subjectivation possible - tout au plus relèvera-ton qu'il est au départ doté d'une bonne stature physique, et doué de fortes capacités intellectuelles. Aussi est-ce précisément la crise de son époque qui décide de son caractère "sans qualités » : c'est parce que son temps est le lieu d'une "pagaille découragée », d'une désorientation généralisée que, dénué de «l'esprit de sérieux » qui lui permettrait d'atteindre le sens des réalités ${ }^{4}$, il sera tout entier tendu vers des possibles eux-mêmes largement indéterminés. De là les jeux constants d'oscillation typiques du personnage : homme du possible, il risque toujours de "sombrer dans une activité toute spleenétique »; mais il est aussi celui qui dispose d'un sens aigu des réalités en tant que possibles ${ }^{5}$; il hait la résignation, il est capable de décisions enthousiastes, mais celles-ci ne mènent jamais nulle part ; il est l'homme de l'essai, de la tentative, mais l'essai retombe toujours, et la tentative revient à son point de départ. Dans une époque où les formes sociales flottent en l'air partes extra partes, sans permettre leur ressaisie subjective et intentionnelle en première personne, alors apparaît un homme sans qualités, lequel se compose, d'abord et avant tout, « de qualités sans homme » :

${ }^{1}$ Sur tout ceci, voir Robert Musil, "L'Allemand comme symptôme», art. cit., p. 367, et «Esprit et expérience », art. cit., p. 115.

${ }^{2}$ Robert Musil, «L'Allemand comme symptôme », art. cit., p. 367.

${ }^{3}$ Selon l'expression de Milan Kundera, L'Art du roman, op. cit., p. 45.

${ }^{4}$ Sur ce thème inspiré des analyses de Sartre, notamment dans L'Etre et le néant, voir le chapitre introductif que consacre Bourdieu à L'Éducation sentimentale dans Les Règles de l'art. Genèse et structure du champ littéraire, Paris, Éditions du Seuil, coll. « Points », 1998, p. 34 sq.

${ }^{5}$ Robert Musil, L'Homme sans qualités, t. 1, op. cit., p. 19. 
Il avait fait ainsi toutes sortes d'expériences et il se sentait capable encore maintenant de se jeter à tout moment dans une aventure sans qu'elle eût nécessairement pour lui le moindre sens, simplement parce qu'elle stimulerait son besoin d'activité. C'est pourquoi il pouvait dire de sa vie, sans exagérer beaucoup, que les événements qui s'y étaient déroulés paraissaient avoir dépendu davantage les uns des autres que de lui-même. Que ce fût dans le combat ou dans l'amour, $\mathrm{B}$ avait toujours suivi $\mathrm{A}^{1}$.

Voilà bien pourquoi, tout comme Debord pourra décrire son époque, en 1961 , comme ce temps où il est possible de «f faire l'essai mais non l'emploi de notre force $»^{2}$, Ulrich songeait «parfois qu'il fût né avec des dons pour lesquels, provisoirement, il n'y avait pas d'emploi » ${ }^{3}$.

\section{Analyse et phénoménologie de la crise chez Musil (et quelques autres) : L'Europe désemparée}

Ne pas avoir d'emploi pour les puissances que l'humanité a développées et accumulées : telle pourrait être la définition de la crise de la civilisation européenne chez Musil. Les éléments épars de la vérité, de l'action efficace et de l'accomplissement du sens et de la valeur existent, mais ils ne trouvent plus de structure susceptible de leur assigner un usage, une inscription sensée dans l'existence. Comme le remarque Maurice Blanchot :

Toute la fabuleuse et ridicule histoire de l'Action parallèle [...] a un sens grave, secrètement dramatique : celui de savoir si la culture peut se donner une valeur ultime ou si elle ne peut que se déployer glorieusement dans le vide contre lequel elle nous protège en le dissimulant ${ }^{4}$.

Musil est à la recherche de cette valeur unifiante, mais ce qui distingue sa démarche des thèmes les plus habituels de la critique du monde moderne est l'absence de toute nostalgie à l'égard d'une époque révolue, soi-disant plus simple et plus consistante. De même, sa critique de la raison calculante ne débouche sur aucune valorisation irrationaliste du chaos psychique ou de la fusion orgiaque avec l'originaire et l'indifférencié : comme on va le voir,

${ }^{1}$ Ibid., p. 176.

${ }^{2}$ Guy Debord, «Critique de la séparation», dans Id., Euvres, Paris, Éditions Gallimard, coll. « Quarto », 2006, p. 544.

${ }^{3}$ Robert Musil, L'Homme sans qualités, t. 1, op. cit., p. 70.

${ }^{4}$ Maurice Blanchot, « Musil », dans Id., Le Livre à venir, Paris, Éditions Gallimard, 1959, p. 202. 
l'Autre État qu'il oppose à l'esprit ratioïde est aussi bien un état de participation à la vie signifiante des choses qu'un jeu inépuisable entre distance et proximité tel que la dualité des sexes le symbolise.

Quel est-il donc le manque que Musil cerne dans l'époque contemporaine, s'il ne saurait être question chez lui ni de la perte d'une Vérité originaire ni d'un appauvrissement de l'authenticité vitale? Dans un essai de 1922, «L'Europe désemparée, ou petit voyage du coq à l'âne ", Musil a esquissé les traits généraux de sa propre version de la Kulturkritik à partir des réactions - mieux: de l'absence de réactions (adéquates) — de la civilisation européenne face au choc de la Première Guerre mondiale :

Nul doute que, depuis dix ans, nous ne fassions de l'histoire mondiale, sous la forme la plus voyante, sans pouvoir, pour autant, l'appréhender. Nous n'avons pas été réellement changés ; un peu d'outrecuidance avant, une vague gueule de bois après; nous étions des citoyens industrieux, nous sommes devenus des meurtriers, des assassins, des voleurs, des incendiaires et ainsi de suite : nous n'avons pas vécu quoi que ce soit pour autant [...]. La guerre nous a paru plus proche du carnaval que de la bacchanale, et la Révolution a fini au Parlement. Nous avons donc été toutes sortes de choses sans changer pour autant, nous avons beaucoup vu et n'avons rien appréhendé ${ }^{1}$.

Musil décrit une condition de l'esprit qui présente maintes analogies avec l'époque actuelle: la malléabilité des êtres, la multiplicité des foyers d'événements, les discontinuités dans le vécu, produisent un état de modification perpétuelle qui échoue à s'inscrire dans une expérience subjective consistante. L'homme qui est exposé à ces modifications est un homme indéfiniment disponible mais dont la versatilité empêche toute maîtrise sur les conditions de son existence. Selon Musil, la crise consiste en l'absence de toute inscription subjective de ce qui nous arrive dans une époque de changements massifs: «Nous ne disposions pas des concepts qui nous auraient permis d'intégrer ce vécu. Ni même, peut-être, des sentiments dont le magnétisme les y aurait aidés $»^{2}$.

La lutte contre le morcèlement de la personnalité et la discontinuité de l'expérience est aussi au centre de l'œuvre d'un contemporain de Musil Franz Kafka. L'homme qui a perdu toute consistance est représenté, dans le Journal, par la figure troublante du « célibataire »:

${ }^{1}$ Robert Musil, «L'Europe désemparée, ou petit voyage du coq à l'âne », dans $I d$., Essais. Conférences, critiques, aphorismes et réflexions, op. cit., p. 135.

${ }^{2}$ Idem. 
Pour celui-ci, il est déjà bien content s'il parvient à maintenir sa personne physique, d'ailleurs pitoyable, à défendre les quelques repas qu'il prend, à éviter l'influence des autres, bref, s'il conserve tout ce qu'il est possible de conserver dans ce monde dissolvant [...]. Le célibataire n'a rien devant lui et, de ce fait, rien non plus derrière. Dans l'instant, cela ne fait pas de différence, mais le célibataire n'a que l'instant. C'est en ce temps que nul aujourd'hui ne peut plus connaître, car rien ne peut être détruit comme ce temps, c'est en ce temps qu'il a échoué ${ }^{1}$.

Le célibataire de Kafka représente une humanité qui a perdu la capacité de s'approprier ses œuvres et de se retrouver en elles :

Tandis que nous étions braqués jusque-là avec notre personne tout entière sur le travail accompli par nos mains, sur ce qui est vu par nos yeux et entendu par nos oreilles, sur les pas faits par nos pieds, nous nous tournons subitement dans la direction opposée, comme une girouette dans la montagne ${ }^{2}$.

Ce n'est pas seulement l'homme laborieux qui se trouve dépossédé de sa consistance par l'extranéation de son œuvre :

Celui qui se présente réellement en bourgeois accompli, celui, donc, qui voyage sur mer dans un bateau avec l'écume devant lui et le sillage derrière, c'est-à-dire tout environné de gros effets [...], lui, ce monsieur et bourgeois n'est pas en moindre danger. Car lui et ses possessions ne font pas un, mais deux, et quiconque brise ce qui les relie le brise du même coup ${ }^{3}$.

L'homme bourgeois, l'homme de la civilisation bourgeoise, découvre que la possibilité de la propriété ne garantit aucunement sa consistance subjective : lui et ses possessions « font deux », leur lien est instable, la propriété privée et éventuellement la richesse ne garantissent plus le développement harmonieux de la personnalité ni la maîtrise des conditions d'existence extérieures et intérieures. Le sujet bourgeois s'étiole, renfermé dans le cercle étroit de possessions qui lui sont devenues étrangères.

Cette condition subjective que Kafka semble évoquer dans son Journal est sans doute très proche de celle que Musil vise par le titre même de son roman inachevé : Der Mann ohne Eigenschaften est l'homme sans qualités, mais aussi et surtout 1'homme sans propriétés. Le "célibataire» au sens kafkaïen qu'est Ulrich est un être dont l'indépendance économique qu'il

${ }^{1}$ Franz Kafka, Journal, trad. fr. M. Robert, Paris, Éditions Grasset, 1954, p. 10-11.

${ }^{2}$ Ibid., p. 11.

${ }^{3}$ Ibid., p. 10-11. 
hérite du mode de vie bourgeois ne correspond plus à une maîtrise authentique de son inscription active dans le monde commun des « citoyens » : les deux visages du Bürger se séparent dans la mesure où les rapports de production qui octroient aux strates bourgeoises un résidu d'autonomie matérielle orchestrent par les mêmes moyens l'hétéronomie universelle sous un régime capitaliste devenu système total, État autoritaire impérialiste et « cage d'acier» (Weber) industrielle, bureaucratique et militaire. Il ne reste au bourgeois que l'oscillation entre la chute dans la prolétarisation - et c'est l'ombre qui pèse sur le minable célibataire de Kafka - et la soumission absurde à la fantasmagorie d'institutions vides de toute signification émotionnelle et rationnelle - et c'est le destin d'Ulrich en tant que secrétaire de l'Action Parallèle. C'est cette condition de dépossession frappant les classes bourgeoises que la guerre a révélé au grand jour, sous la forme d'une béance irréductible qui se creuse sous les structures de l'existence.

Par conséquent, la crise n'est pas vue comme l'éloignement illégitime d'une unité organique ; la guerre a sans doute révélé une vérité définitive que l'homme n'a pas de fondement univoque et stable: «L'homme, depuis 1914, s'est révélé une masse étonnamment plus malléable qu'on ne l'admettait généralement ${ }^{1}$. Il s'agit d'assumer jusqu'au bout cette révélation sans renoncer pour autant à s'interroger sur les conditions de la maîtrise subjective de la condition humaine. Maîtriser subjectivement la multiplicité et le devenir signifie pouvoir inscrire leurs effets dans la construction d'une personnalité et de relations sociales consistantes.

La crise de la civilisation se manifeste donc comme impasse du pouvoir de construire et de donner forme cohérente aux éléments flottants du savoir, du sentiment et du monde extérieur. La description de la fragmentation et de l'incohérence du monde contemporain est chez Musil somme toute assez traditionnelle - elle se rapproche, par exemple en ce qui concerne la critique de l'épistémologie positiviste et de l'idéal des «faits », des Considérations inactuelles de Nietzsche :

L'abondance de faits devint surabondance, et la recherche historique, confrontée à cette pléthore, fut condamnée à se faire toujours plus pragmatique et plus exacte : le résultat fut un vrai cauchemar, une montagne de fait grossissant d'heure en heure, un gain de savoir et une perte de vie, un échec psychique $^{2}$.

${ }^{1}$ Robert Musil, «L'Europe désemparée, ou petit voyage du coq à l'âne », in $I d$., Essais. Conférences, critiques, aphorismes et réflexions, op. cit., p. 140.

${ }^{2}$ Ibid., p. 143. 
Mais il n'y a chez Musil aucune apologie de l'innocence ou du non-savoir pré-moderne. En fait, ce qui manque à l'homme contemporain est la possibilité même de se servir tant des connaissances modernes que des valeurs archaïques : «Les concepts qui pourraient ordonner la vie qui nous entoure font défaut. Les faits du passé, les faits des diverses sciences et les faits de la vie nous submergent pêle-mêle $»^{1}$.

L'absence de concepts organisateurs caractérise tant l'effondrement des mythes optimistes du XIX ${ }^{\mathrm{e}}$ siècle et de la Belle Époque que les tentations nostalgiques et traditionnalistes: "Tout ce qui relève de l'esprit est livré désormais au chaos. Par tradition et sans plus bien savoir pourquoi, on combat l'esprit fondé sur les faits et les nombres, mais sans avoir à lui opposer que la pure négation $»^{2}$.

Une autre analogie frappante entre la situation historique de Musil et la nôtre est précisément ce manque accablant de conséquences pratiques qu'on pourrait tirer du malaise persistant au sein de la civilisation :

Quand on proclame, en effet - et qui ne fait plus ou moins chorus ? - , que notre temps manque de synthèse, de culture, d'esprit religieux ou communautaire, cela ne dépasse guère l'éloge du « bon vieux temps », personne n'étant en mesure d'expliquer ce que devraient être aujourd'hui une culture, une religion ou une communauté pour peu qu'on voulût vraiment intégrer dans sa synthèse les laboratoires, les avions et le dinosaure social, au lieu de les juger simplement dépassés ${ }^{3}$.

Cette incapacité à imaginer des débouchés réels susceptibles de soutenir la rigueur de la critique implique nécessairement la fragmentation et l'éclectisme idéologique :

Notre temps abrite côte à côte et dans la plus totale dysharmonie tous les contraires : individualisme et sens communautaire, aristocratisme et socialisme, pacifisme et bellicisme, exaltation de la culture et industrie de la civilisation, nationalisme et internationalisme, religion et sciences de la nature, intuition et rationalisme, et bien d'autres encore [...]. Même à ne la considérer que du dehors, on devine que cette typologie antithétique - cette façon d'exposer les problèmes par couples d'opposés, cette multiplication des « ou bien... ou bien...» - dénote un travail intellectuel insuffisant [...]. Dans la réalité pratique, notre paysage intellectuel offre un collectivisme de chapelles poussé à l'extrême. Chaque communauté de lecteurs a son écrivain, le parti

${ }^{1}$ Ibid., p. 148.

${ }^{2}$ Idem.

${ }^{3}$ Idem. 
des agriculteurs et celui des ouvriers ont chacun sa philosophie ; il y a peutêtre en Allemagne une centaine de maisons d'édition dont chacune a son cercle de lecteurs plus ou moins étroitement liés par une sensibilité commune ; le clergé a son réseau, mais les anthroposophes ont leurs millions et les universités leur crédit [...]. C'est une maison de fous babélique ; de ses milliers de fenêtres, des milliers de voix, de pensées, de musiques différentes agressent en même temps le passant; dans ces conditions, il est clair que l'individu devient une arène où s'affrontent des motifs anarchiques et que la morale, à l'instar de l'esprit, se désagrège ${ }^{1}$.

Finalement, la capitulation éthique et politique devant la puissance nue des formes vides qui continuent à «fonctionner» est le débouché naturel de la désorientation générale :

Un symptôme très caractéristique de la catastrophe a été en même temps l'expression d'une certaine situation idéologique : c'est notre complète inertie à l'égard des groupes de spécialistes chargés de la machine de l'État; nous avons été pareils à ces voyageurs de wagons-lits qui ne se réveillent qu'au moment de la collision. Un tel laisser-faire n'est pas le fait seulement des « citoyens pensants » à l'égard des « organes agissants » de l'État, mais aussi celui des idéologies concurrentes : elles aboient, mais ne mordent pas²

L'abdication devant les experts et les technocrates relève avant tout d'un «manque d'organisation intellectuelle»: «Les problèmes idéologiques, dans leur aspect confus et creux, étaient réservés aux "beaux esprits", alors que les pouvoirs politiques réalistes avaient au moins sur eux l'avantage d'une certaine capacité juridique $»^{3}$.

Musil refuse toute posture «humaniste» et sentimentale: les tendances catastrophiques de l'époque ne peuvent être critiquées qu'à partir d'une capacité d'organisation supérieure à celle des forces qui poussent vers le désastre. Ce n'est pas l'idylle nostalgique qui peut représenter une alternative aux puissances du capital, de l'État et de la technologie, mais une plus grande capacité de maîtrise et d'organisation des possibles et des réels :

Toutes les valeurs chancèlent [...]. N'allons pas croire pour autant qu'une simple réparation, une restitutio in integrum [...] puisse améliorer notre situation; car c'est moins l'idéalité qui a fait défaut que ses simples et nécessaires prémisses [...]. La solution ne se trouve ni dans l'attente d'une

\footnotetext{
${ }^{1}$ Ibid., p. 149.

2 Ibid., p. 150.

${ }^{3}$ Ibid., p. 150-151.
} 
idéologie nouvelle, ni dans le combat des idéologies aujourd'hui opposées ; elle est dans la création des conditions sociales capables d'assurer aux efforts idéologiques une stabilité et un tirant d'eau suffisants. Ce qui nous manque, ce ne sont pas les contenus, c'est la fonction ! ${ }^{1}$

La fonction : à savoir, le lien subjectif entre l'esprit et ses contenus, un lien qui doit être susceptible de subjectiver ces contenus en les incorporant dans une structure organique de la personnalité :

L'énorme problème d'organisation qui s'impose du même coup consiste à ne pas abandonner la confrontation et la mise en relation des facteurs idéologiques au hasard, mais bien à les favoriser. Cette fonction indispensable n'existe aujourd'hui que dans le domaine des sciences, du pur entendement; dans le domaine de l'esprit, les créateurs eux-mêmes n'en ont pas compris la nécessité ${ }^{2}$.

L'entendement scientifique tel que Musil l'interprète - un entendement réducteur, analytique, opérationnel - opère des synthèses, organise des contenus et des expériences. Mais ces synthèses et ces organisations ne s'incorporent que très superficiellement à la vie subjective - elles existent comme des choses extérieures :

Le cours habituel de nos pensées va d'une pensée à l'autre et d'un fait à l'autre en éliminant le moi, nos pensées comme nos actes ne passent pas par notre moi. Aussi bien est-ce là l'essence de notre objectivité : elle relie les choses entre elles et, même quand elle les met en rapport avec nous ou comme dans la psychologie - nous choisit pour son objet, elle le fait en éliminant l'élément personnel [...]. Dès lors, l'ordre que fonde l'objectivité ne peut valoir que pour les choses, et non pour les humains ${ }^{3}$.

À cette synthèse objective Musil oppose une pensée « baignant plus profondément dans le sentiment, une relation plus personnelle avec l'expérience » ${ }^{4}$, telle qu'elle se manifeste dans la littérature mystique et dans la sagesse, dans les œuvres d'Eckhart, Confucius ou Emerson :

Cet ordre de l'art, de l'éthique et de la mystique, c'est-à-dire du monde du sentiment et de l'idée, opère, il est vrai, un travail de comparaison, d'analyse

\footnotetext{
${ }^{1}$ Ibid., p. 152.

${ }^{2}$ Ibid., p. 153.

${ }^{3}$ Ibid., p. 154.

${ }^{4}$ Idem
} 
et de synthèse, en quoi il est rationnel et apparenté par essence aux plus puissants instincts de notre temps; mais il n'est pas en opposition avec l'âme ; il a sa fin propre : non pas cette univocité qui réduit l'éthique à une morale ou le sentiment à une psychologie causale, mais un regard d'ensemble sur les raisons, les connexions, les restrictions, les fluctuations de sens de nos motifs et de nos actes - une glose de la vie ${ }^{1}$.

Ce mode de la pensée - dont les procédures sont rationnelles, mais dont les finalités relèvent moins du savoir que de la qualité de l'expérience subjective - est devenu incapable d'organiser les contenus culturels et psychiques de l'époque contemporaine: "Ce qui nous reste aujourd'hui de véritable éthique, on le trouvera, sous une forme très insuffisante, dans les arts, les essais et dans le chaos des relations privées $»^{2}$.

Pourtant, c'est précisément de ces micro-phénomènes où s'est conservée la sagesse qu'il faut repartir afin de ne pas laisser les tâches de l'organisation du monde à la seule sphère de l'entendement ou ratioïde.

\section{Du (non-)ratioïde. Une crise épistémologique et son renversement éthique}

Le lieu privilégié du diagnostic musilien quant au désarroi, au découragement dominant l'époque, est le domaine de la vie intellectuelle, en particulier le champ scientifique (Musil était ingénieur de formation).

Partagée entre « scrupulosité bornée » et « superficialité effrénée » ${ }^{3}$, la rationalité scientifique dominante se révèle n'être pas à la hauteur des enjeux épistémologiques et éthiques de son temps. Elle pèche en fait par deux côtés. Misant tout sur la croyance aux faits, la raison scientifique se définit d'abord par l'empirisme étroit, rétréci, de «l'expérience triviale»: «Ce qui caractérise, ce qui détermine notre situation intellectuelle, écrit ainsi Musil, c'est [...] la pléthore des contenus, l'hypertrophie de la science des faits $\gg{ }^{4}$. Pour autant, de l'autre côté, ce positivisme obtus et répétitif est comme compensé, complété par un recours permanent à l'intuition irrationnelle, à la

\footnotetext{
${ }^{1}$ Ibid., p. 156.

${ }^{2}$ Ibid., p. 155.

${ }^{3}$ Robert Musil, «Esprit et expérience », art. cit., p. 115.

${ }^{4}$ Ibid., p. 104, 102. Un tel constat fonde par ailleurs la critique du positivisme commune, de Marcuse à Adorno, à toute l'École de Francfort, et il s'origine dans l'analyse des rapports entre abstrait et concret menée par Marx au $\S 3$ de sa préface aux Grundrisse (1857), « La méthode de l'économie politique ».
} 
vérité, réputée infalsifiable, du sentiment, à quelque chose de l'ordre de la Schwärmerei - de là la critique que Musil oppose à ce que l'on pourrait appeler, cette fois avec la Préface de la Phénoménologie de l'Esprit, l'oppression du concept différenciant dans la restauration du sentiment de l'essence :

Mais que toute la richesse de l'intuition aboutisse finalement à ceci : [...] que l'on se montre incroyablement sceptique in ratione [...], mais incroyablement crédule à l'égard de tout ce qui vous passe par la tête $[\ldots]$ : voilà le portrait clinique de l'esprit aveuli par les jouissances trop prolongées de l'intuition, du bel esprit de notre temps ${ }^{1}$.

Cette situation ambiguë et calamiteuse caractérise ce que Musil nomme le « ratioïde ». Le mot est chargé de désigner l'état de morcellement actuel du champ du savoir, «véritable chaos de pensées, de sentiments et de forces directrices parfaitement contradictoires», dessinant une «situation idéologique [...] extrêmement particularisée, voire individualisée $»^{2}$. Dans ces conditions, le problème est double. D'une part, il n'est plus possible de subsumer cet ensemble disparate dans une pensée qui le viserait comme totalité signifiante: "Aucune initiative ne peut pénétrer le corps social sur une longue distance, ni recevoir le contrecoup de la totalité de celle-ci ». Réciproquement, il n'est plus donné à l'homme de conférer à ces formes du savoir en morceaux un contenu intentionnel en rapport avec les exigences de son existence et de son épistémè : «Des parties individuelles [...] font l'objet de choix non moins individuels » et, concrètement, l'organisation et l'orientation du champ du savoir devient l'apanage d'experts ${ }^{3}$. En tout ceci, le ratioïde apparaît comme une réponse à un désir de sécurité infantile, de puérile réassurance face aux bouleversements épistémologiques de l'époque ; mais, on le voit, il semble ne pas même être en mesure de tenir les promesses de ce combat d'arrière-garde, tout en manquant complètement, du reste, le sens authentique du rapport de l'homme à la connaissance.

Bref, le ratioïde se montre tout à fait incapable d'assumer la crise historique de la raison fondationnaliste ; et il ne peut davantage faire droit aux phénomènes irrationnels qui, de tout temps, trouent la connaissance rationnelle. C'est ce double échec que met en évidence Gargani dans un commentaire inspiré de Törless :

${ }^{1}$ Ibid., p. 112.

${ }^{2}$ Robert Musil, « L'Allemand comme symptôme », art. cit., p. 361.

${ }^{3}$ Voir, respectivement, Robert Musil, «Esprit et expérience», art. cit., p. 115, et «L'Allemand comme symptôme », art. cit., p. 61. 
Törless est le miroir d'une crise des fondements, de la mise en question d'une connaissance absolue [...] et de la conscience d'une réalité irrationnelle [...]. L'expérience existentielle et intellectuelle de Törless atteint son point culminant [...] dans la conscience que la rationalité, la moralité et le langage traditionnels sont incapables de comprendre cette réalité irrationnelle faite d'expériences incomplètes [...] propres à cet être incomplet [...] qu'est l'homme ; une réalité irrationnelle qui échappe à l'homme dans la sphère ratioïde $[\ldots]$, c'est-à-dire dans le domaine d'une pensée logicisante, monotone, invariante, dominée par le pouvoir de la répétition ${ }^{1}$.

Ainsi s'explique, au total,

l'intellectualisme, au sens péjoratif du mot, la mode actuelle de la précipitation intellectuelle, le flétrissement prématuré des pensées. Par le fait que nous cherchons la profondeur avec nos pensées et la vérité avec nos sentiments; et que, faute de voir cette interversion, nous sommes à tout moment déçus ${ }^{2}$.

La clef de l'explication réside somme toute dans la mainmise du régime ratioïde sur la pensée : il a la charge de conférer un semblant de sens à une époque incapable de penser sa spécificité épistémologique, tout comme l'essence même du travail de la connaissance, et qui s'en remet, en désespoir de cause, à la confusion de la pensée et des sentiments, du concept et du vécu, de la raison et de l'intuition.

Or, si cette situation est bien porteuse d'un grave péril, il est cependant peut-être possible de la surmonter, à la condition, éthique, de se faire « une âme plus ferme $»^{3}$ — où l'on retrouve, sur fond d'un constat proche de celui de Lukács (et, en amont, de Weber), l’alternative husserlienne : « Héroïsme ou barbarie ». Dans cette perspective, le projet fondamental de Musil est en fait de parvenir à procéder au renversement de l'interversion du sentiment et de la pensée; ou encore, en d'autres termes, qui sont ceux-là mêmes de l'auteur, de retrouver le fondement de la causalité — laquelle « cherche la règle à travers la régularité » — dans la motivation qui, en vérité, l'institue, puisqu'elle délivre « l'impulsion qui pousse à agir, à sentir, à penser dans ce sens $\gg{ }^{4}$. On le comprend : s'il ne peut s'agir ici de sauter à pieds joints dans le cercle de l'irrationnel, la crise de la raison impose - comme chez Kant, comme chez Husserl — de procéder à sa critique, mais afin de la recouvrer

\footnotetext{
${ }^{1}$ Aldo Giorgio Gargani, L'Étonnement et le hasard, op. cit., p. 138.

${ }^{2}$ Robert Musil, « Esprit et expérience », art. cit., p. 114.

${ }^{3}$ Ibid., p. 102.

${ }^{4}$ Ibid., p. 109.
} 
sous une forme nouvelle, enfin créatrice celle-ci ; reconquête qui en ultime analyse impose une transformation de soi, l'adoption d'une nouvelle « attitude éthique ». La critique du ratioïde aboutit donc à l'exigence d'une réinvention du lien concept/vécu, à une ré-articulation de ce qui a été malheureusement interverti. Soit « un nouveau rapport entre l'intellect et le sentiment $»^{1}$ qui doit permettre d'unifier, pour la reconquérir subjectivement, par le souffle d'une intention signifiante, une raison actuellement impersonnelle, et brisée en mille fragments. Voici sur ce point le passage essentiel des Essais :

La pensée purement rationnelle, qui semble absolument étrangère au sentiment, peut aussi être stimulée par des états d'âme plus intenses. À combien plus forte raison la pensée que nous appelons ici non ratioïde, dont la force de pénétration et la vitesse de propagation intérieure dépendent justement de la vitalité des mots, de cette sorte de nuage de pensée et de sentiment qui enveloppe l'insignifiant noyau conceptuel [...]. Le monde dans lequel nous vivons $[\ldots]$ n'est que le succédané d'un autre monde avec lequel la vraie relation s'est perdue. On sent parfois que rien de tout cela n'est essentiel; pour quelques heures, ou quelques jours, tout cela fond au feu d'un autre comportement envers les autres et le monde $[\ldots]$. Telle est l'atmosphère des états philosophiques créateurs ou éclectiques ${ }^{2}$.

De façon générale, on tiendra que le projet épistémologique de Musil consiste en une refondation des rapports entre les exigences de l'existence et celles de l'épistémè, vers l'invention d'une rationalité qui, par-delà les apories du ratioïde, entre logique et existence, se réapproprierait son pouvoir créateur. Un tel travail trouve sa sphère d'expérimentation privilégiée dans le domaine littéraire, en particulier sous la forme de l'essai, là où domine «l'expérience vécue » et où « au concept rigide se substitue la représentation créatrice $»^{3}$ - on voit combien Gargani avait raison de dire que la crise épistémologique implique chez Musil la reconsidération des rapports entre éthique et esthétique. Pour autant, encore une fois, cette réévaluation des pouvoirs de la raison menée, dans le champ de l'esthétique, sous condition d'une nécessité éthique, ne revient pas à laisser libre cours aux flottements de l'âme - c'est bien la «fermeté » de celle-ci que l'on recherche ; autrement dit: «Le débat encore stérile entre la pensée scientifique et les exigences de

\footnotetext{
${ }^{1}$ Aldo Giorgio Gargani, L'Étonnement et le hasard, op. cit., p. 140, ou p. 148.

${ }^{2}$ Robert Musil, «Esprit et expérience », art. cit., p. 111.

${ }^{3}$ Ibid., p. 106.
} 
l'âme ne peut être tranché que par un "plus", un plan, une orientation de travail, une nouvelle évaluation de la science comme de la littérature $»^{1}$.

Résumons tout ceci d'un trait, en joignant deux formules musiliennes : la découverte d'une "mathématique morale plus fine » ne peut aller sans un « amour humain des sciences $»^{2}$; et la réciproque n'est pas moins vraie.

Nous nous en voudrions de ne pas montrer, en conclusion de ce point, que le personnage de Törless peut à bon droit être envisagé comme cet « ego expérimental » qui, logé exactement au point de croisement décrit à l'instant, éprouve, parfois dans le désarroi, ses conséquences existentielles finales. Sujet aux affres de son âge, Törless s'interroge : "Qu'est-ce donc que cette qualité particulière que je possède ? $»^{3}$. Cette spécificité, encore indéterminée, lui a été révélée à l'occasion d'une expérience quasi-mystique, proche de ce que Musil appelle "l'autre état», où se brouillent les frontières du moi et du monde, où se montre enfin ce qu'il y a « en nous [...] de plus fort, de plus grand, de plus beau, de plus passionné, de plus obscur que nous ${ }^{4}$. Le point remarquable est que cette expérience existentielle limite se répète pour peu à peu trouver sons sens achevé, sa forme personnelle - lorsque Törless s'avise de l'existence des nombres irrationnels, autrement dit: des trous d'irréalités qui grèvent la connaissance la plus rationnelle du réel, de l'abîme sur laquelle elle repose. D'un seul coup, les mathématiques passionnent le lycéen : à leur endroit, " il éprouvait [...] un soudain respect : d'aride matière à mémorisation, elles étaient devenues d'un coup pour lui problème vivant $»^{5}$. L'expérience n'est cependant pas paisible: car cette rencontre où se rejoue le rapport de "l'expérience vivante» et de la « connaissance» ne va pas sans mettre en lumière le fond d'inquiétude sur lequel se détachent toujours «nos tâches quotidiennes " ${ }^{6}$. C'est sans doute pour limiter ce pouvoir d'inquiétude qu'un professeur conseille à Törless de lire la Critique de la raison pure ${ }^{7}$; mais l'ouvrage déçoit le jeune homme : à la recherche d'une nouvelle alliance du sentiment et de la pensée, Kant lui apparaît fatalement comme symbole de ce que Musil décrira plus tard sous le nom de « ratioïde ».

${ }^{1}$ Ibid., p. 116-117.

${ }^{2}$ Voir, respectivement, Robert Musil, «L’Europe désemparée », art. cit., p. 140, et L'Homme sans qualités, op. cit., p. 46.

${ }^{3}$ Robert Musil, Les Désarrois de l'élève Törless, trad. fr. P. Jaccottet, Paris, Éditions du Seuil, coll. « Points », 2012, p. 150.

${ }^{4}$ Ibid., p. 151. On lira l'épisode de l'expérience mystique du parc, p. 99 sq.

${ }^{5}$ Ibid., p. 123.

${ }^{6}$ Ibid., p. 104.

${ }^{7}$ Ibid., p. 127 sq. 
Ce sera donc seulement par une conversion éthique, par la découverte de « sa véritable personnalité », la formation et l'affirmation de son « caractère ${ }^{1}$, que Törless parviendra à réconcilier, pour son propre compte, l'existence et la raison: en faisant droit à l'irréalité, et donc aux possibles, qui hypothèquent toujours la clôture sur soi du réel-rationnel. Ce qu'il exprime devant ses professeurs atterrés en un final splendide :

Une pensée qui peut avoir traversé depuis longtemps notre cerveau ne devient vivante qu'au moment où quelque chose qui n'est plus de la pensée, qui ne relève plus de la logique, s'y ajoute : de sorte que nous éprouvons sa vérité indépendamment de toute preuve, comme si elle avait jeté l'ancre dans la chair vivante, irriguée de sang... Une grande découverte ne s'accomplit que pour une part dans la région éclairée de la conscience ; pour l'autre part, elle s'opère dans le sombre humus intime, et elle est avant tout un état d'âme à la pointe extrême duquel s'ouvre comme une fleur ${ }^{2}$.

\section{L'《 autre état »}

La culture positiviste se fonde sur la pensée ratioïde ; l'œuvre de Musil vise à esquisser une organisation différente des phénomènes de l'esprit — tout comme Gaston Bachelard dans un contexte culturel à la fois différent et proche, l'écrivain autrichien cherche un principe de complémentarité entre l'âme et la précision, la patience du concept et la prégnance du sens. Cette complémentarité s'oppose à la réduction de la raison à des procédures de calcul :

Il existe un état humain qu'une différence fondamentale oppose à celui de la connaissance, du calcul, de la poursuite des buts, de l'évaluation, de la contrainte, de la convoitise et des craintes viles. Il est difficile à caractériser. Dans toutes ses caractérisations par la bonté, l'amour, l'irrationalisme, la religiosité, ici combattue, il y a une part de vérité : mais pour saisir la vérité entière, il nous manque encore la pensée adéquate. J'aimerais l'appeler simplement «l'autre état $»^{3}$.

Du point de vue de l'autre état, "penser et convoiter apparaissent ne faire qu'un » ${ }^{4}$. La caractérisation de l'autre état révèle que ce mode «nocturne»

${ }^{1}$ Ibid., p. 62, 130 .

${ }^{2}$ Ibid., p. 231.

${ }^{3}$ Robert Musil, « L'allemand comme symptôme », art. cit., p. 374.

${ }^{4}$ Idem. 
de la pensée représente pour Musil le pivot d'une critique de toute relation au monde fondée sur l'appropriation et la possession : dans l'état ordinaire

une fine ligne, une espèce de tige, lie la personne à son objet en ne prenant appui, d'un côté comme de l'autre, que sur un point, sans rien concerner autour ; l'image est valable pour le connaître comme pour le vouloir et, de fait, on les a souvent condamnés ensemble comme les deux faces d'un même $\mathrm{mal}^{1}$.

Dans l'autre état, les choses et les êtres sont examinés " avec sympathie », avec amour, ce qui change leur manière d'être en fonction de leur rapport au sujet : la relation d'appropriation sur laquelle se fonde l'attitude productive — économique et technoscientifique — du sujet moderne est déjouée. Le sujet y fait l'expérience de la déprise et de la dés-appropriation :

Nous avons de très nombreuses descriptions de cet autre état. Toutes pourraient bien avoir en commun une séparation moins marquée entre le moi et le non-moi et un certain renversement de leur rapport (égoïsme et mesures). Alors que, dans l'état ordinaire, le moi prend possession du monde, dans l'autre état, le monde afflue vers le moi ou se confond avec lui ou le porte etc. (passif au lieu d'actif). On a part aux choses (comprend leur langage). Dans cet état, l'acte de compréhension n'est pas impersonnel (objectif), mais se manifeste de façon personnelle comme un accord parfait entre sujet et objet ${ }^{2}$.

L'autre état représente le dépassement, ou l'explosion, de l'anthropologie bourgeoise et de sa crise interne: en lui, la déprise, l'interruption de la relation propriétaire, coïncide immédiatement avec l'appropriation intensive du sens des choses, un sens qui précède, ou néglige tout simplement, la séparation entre le sujet et l'objet et la manipulation que le premier exerce sur le second :

Le contraire de l'objectivité est une intensification du moi ou de l'objet, mais non une subjectivité [...]. On peut dire que là, le moi, comme le monde, cessent d'être des choses. Il s'agit d'une autre façon d'évaluer. L'opposition altruiste-égoïste perd son sens ; comme celle entre bon et mauvais. On peut leur substituer le couple accroissement/diminution. Également : à ce qui est utile se substitue ce qui accroît. Cela implique encore le dépouillement de

\footnotetext{
${ }^{1}$ Idem.

${ }^{2}$ Ibid., p. 375.
} 
toute petitesse. Dans la forme contemplative, souvent, le sentiment de s'abîmer, de se dissoudre, d'être porté ${ }^{1}$.

L'autre état est différent tant de la relation propriétaire que de la fusion orgiastique - la proximité entre le sujet et les choses y coïncide avec une distance respectueuse qui n'annule jamais l'écart entre l'émotion et son monde. Ce paradoxe s'exprime clairement dans l'extase qui introduit Ulrich et Agathe dans la sphère de l'autre état: "L'“Impossible" qui flottait déjà comme une présence quasi physique autour d'Agathe et d'Ulrich se reproduisit, et cela, en vérité, sans que quoi que ce fût se produisît $»^{2}$.

L'extase réconcilie l'unité et la différence, la proximité et la distance. Le frère et la sœur découvrent la communion ontologique de leurs natures individuelles : "Il semblait que par l'harmonieux partage de leur stature fraternelle leurs corps montassent d'une racine unique $»^{3}$.

Pourtant, le désir d'unité que représente la relation fraternelle n'est jamais susceptible d'annuler la distance infranchissable dont le symbole est la dualité des sexes :

Ils subissaient, en bravant autrui, ce besoin commun de se délivrer enfin de la tristesse du désir, mais [...] une exigence plus forte encore leur commandait le calme, et ils furent incapables de se toucher à nouveau. Ils voulurent essayer, mais les gestes de la chair leur étaient devenus impossibles et ils ressentaient comme un avertissement ineffable qui n'avait rien à voir avec les commandements de la morale. Il semblait que, de ce monde où l'union, bien qu'encore presque irréelle, est plus parfaite, et qu'ils venaient de savourer comme dans une métaphore exaltée, un commandement plus élevé les eût atteints ${ }^{4}$.

C'est pourquoi l'expérience amoureuse ne saurait plus se consommer désormais que par la distance irréductible introduite par la parole — dans des « dialogues d'amour» où le rapprochement et l'éloignement sont infiniment variés et réélaborés comme un thème mythologique inépuisable : "Ainsi s'ébaucha de nouveau un mouvement qui les fit pencher l'un vers l'autre et les sépara l'un de l'autre $»^{5}$.

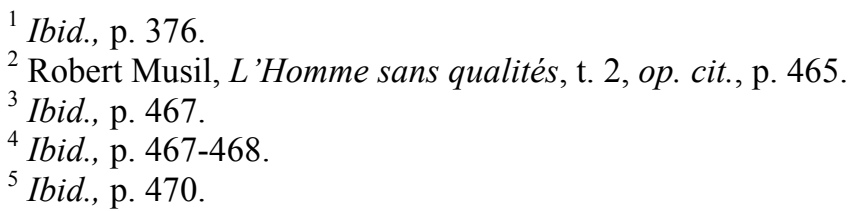


L'oscillation qui sépare et unit Ulrich et Agathe passe sans discontinuité dans la bascule entre le sujet et les choses qui caractérise l'autre état :

Les formes et les couleurs qui s'offraient à eux étaient dissoutes, sans fond, et néanmoins frappantes comme un bouquet de fleurs sur une eau sombre $[\ldots]$ L'impression relevait autant du domaine défini de la perception et de l'attention que de celui, imprécis, du sentiment; cela même la faisait flotter entre le dedans et le dehors comme le souffle qu'on retient flotte entre l'inspiration et l'expiration [...]; elle les contraignit à garder leurs distances, bien que leur sensibilisation durât et pût laisser croire que s'était soudain modifié le tracé des frontières qui les séparaient l'un de l'autre comme elles les séparaient du monde ${ }^{1}$.

\section{De l'essai. Expérience littéraire et cas éthique de « l'autre état»}

Musil ne craint pas d'écrire que l'idée de «l'autre état» vaut pour condition d'une « véritable éthique »; mais il ajoute aussitôt que c'est seulement sous une forme insuffisante qu'il se dévoile aujourd'hui, par approximation, «dans les arts, les essais et le chaos des relations privées », amoureuses notamment. L'erreur - à y succomber, elle nous empêcherait définitivement de nous approcher d'une telle éthique - serait de penser qu'en ces sphères domine le déchaînement des passions: selon Musil, il n'en est rien. À l'inverse, c'est selon lui justement au moment où il devient urgent d'exprimer le nœud - complexe mais investi d'un pouvoir régénérateur du concept et du vécu qu'il devient également nécessaire d'en appeler à un maximum de discipline. Discipline individuelle, certes, mais pas seulement, puisque, comme l'auteur le précise : «Une époque qui aura cru pouvoir faire l'économie de ce travail et de cette discipline ne sera jamais capable de résoudre les grands problèmes de l'organisation du monde $»^{2}$. Musil relie donc explicitement la question esthétique de l'essai littéraire à la dimension de l'éthique; et si cette dernière semble bien faire signe, avec la notion de discipline, vers le problème de la transformation subjective, on pressent aussi qu'elle se dépasse, cette fois avec la notion d'organisation, vers le domaine de la politique proprement dite. En tentant, à la suite de Musil, de rapporter cette notion d'essai (Versuch) au concept d'expérience (Erfahrung ou Erlebnis), c'est la conception de la subjectivité qui s'infère de l'œuvre de Musil que nous aborderons pour terminer.

${ }^{1}$ Ibid., p. 472.

${ }^{2}$ Sur tout ceci, voir Robert Musil, « L’Europe désemparée », art. cit., p. 155, 156. 
Situé au point de jonction « du savoir, de la science » — du concept et « de la vie et de l'art » - du vécu —, la forme littéraire autonome de l'essai est définie par Musil d'une formule aphoristique : l'essai présente « le comble de la rigueur accessible dans un domaine où le travail exact est impossible $»^{1}$. Autrement dit, s'affrontant à la singularité, à l'idiosyncrasie de « faits qui ne se prêtent pas à une observation généralisée », il appartient à l'essai de tenter, autant que faire se peut, de rétablir « un ordre », un véritable "enchaînements de pensée », une systématicité ${ }^{2}$. C'est en ce sens qu'il est capable de prendre en charge ce que Törless appelait les «pensées vivantes », c'est-à-dire, on l'a vu, ces réflexions « d'ordre sentimental » qui ne tombent pas seulement sous le coup du critère de vérité, tant elles « interpellent » intimement le sujet de la pensée ; et l'on comprend son importance lorsque l'on s'avise qu' « il existe des réflexions qui n'agissent, au fond, que par cette voie ». Si bien que l'essai, comme forme esthétique, a au total à prendre en charge ce qui, dans le domaine du travail de la pensée, excède l'idéal de la connaissance pour rejoindre le problème — que l'on dira éthique - de « la transformation de l'homme $»^{3}$.

Une telle détermination de l'éthique, fondée sur l'idée d'une transformation subjective, d'une modification de soi par soi, n'est pas étrangère, on le sait, au travail que le dernier Foucault a consacré aux modalités antiques de la subjectivation morale : l'éthique renvoie dans ce cadre à l' " ascèse », au « rapport à soi » que le sujet se doit d'exercer afin de se faire sujet de tel ou tel code moral ${ }^{4}$. Retrouvant le sens originel de l'éthique comme êthos, c'est alors la sphère de la conduite subjective (ce que Weber, avant Lukács, nommait Führung, Lebensführung) qui vient avec Foucault au centre de l'investigation.

Notons-le, la convocation de ce dernier auteur dans le présent contexte se justifie d'au-moins deux façons. D'une part, parce que le concept de l'éthique qu'il propose repose également sur une tentative de ré-articulation du savoir et du vécu, sur l'idée que l'assimilation de la connaissance suppose et implique une pratique active de transformation du sujet par lui-même; d'autre part, et surtout, parce que cette étape du parcours foucaldien s'ordonne à une refonte corrélative du concept d'expérience, dont le sens ultime

\footnotetext{
${ }^{1}$ Robert Musil, « De l'essai », dans Id., Essais. Conférences, critiques, aphorismes et réflexions, op. cit., p. 334.

2 Ibid., p. 335, 338.

${ }^{3}$ Sur tout ceci, ibid., p. 337.

${ }^{4}$ Michel Foucault, L'Usage des plaisirs, Paris, Éditions Gallimard, coll. « Tel», p. 16,40 .
} 


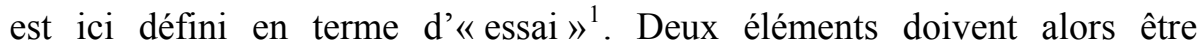
soulignés. D'un côté, l'essai, dans cette perspective, représente l'expériencelimite entreprise par un sujet au nœud de «domaines de savoir, types de normativité et formes de subjectivité » imposés par les formes objectives de l'expérience ${ }^{2}$, le sujet découvrant en ce lieu soit les limites de son assujettissement, soit la possibilité de leur re-subjectivation à nouveaux frais - c'est dans ce seul dernier cas qu'il convient de parler d'expérience-limite au sens strict du terme. Or - d'un autre côté - il faut se souvenir que cette détermination de l'expérience éthique (comme essai, travail à la limite) est chez Foucault d'origine littéraire : c'est elle qu'il trouve chez Bataille et Blanchot et qu'il explore pour son propre compte, notamment dans ses recherches consacrées à l'expérience de la folie ${ }^{3}$. Aussi pourrait-on soutenir que l'expérience éthique - désormais comprise comme une tentative, un essai pour se porter aux limites de notre pensée et de notre existence, afin d'apprendre à penser et à vivre autrement, à inventer par épreuves une autre forme $d u$ soi -, correspond à la reprise apaisée d'une expérience-limite qui, auparavant, entre littérature et folie, débouchait seulement sur une déprise radicale de soi-même synonyme de destruction de toute forme subjective ${ }^{4}$. Ainsi passe-t-on avec Foucault, des années soixante aux années quatrevingts, du thème de l'«absence d'œuvre » à celui de «l'existence comme œuvre d'art », ou encore de la sphère du sublime à celle du beau : par le biais d'une prise en compte de la discipline, du protocole que réclame l'expérience-limite du sujet dans son risque même (du point de vue de sa

${ }^{1}$ Ibid., p. 16 : «Le discours philosophique » a le «droit d'explorer ce qui, dans sa propre pensée, peut être changé par l'exercice qu'il fait d'un savoir qui lui est étranger »; et c'est à ce titre que « l'essai », compris « comme épreuve modificatrice de soi-même dans le jeu de la vérité », pourra apparaître comme « le corps vivant de la philosophie ». Sur le lien du concept et de l'expérience, la référence à Canguilhem est chez Foucault décisive, voire exemplairement Michel Foucault, «La vie: l'expérience et la science », dans Id., Dits et écrits, t. 2, Paris, Éditions Gallimard, coll. « Quarto », 2001, p. 1582-1595; les notions d'essai et d'épreuve engagent du reste le tout de la pensée de Canguilhem quant à la normativité vitale, voir par ex. Georges Canguilhem "Le Normal et la pathologique », dans Id., La Connaissance de la vie, Paris, Librairie philosophique J. Vrin, coll. "Bibliothèque des Textes Philosophiques », 2006, p. 201.

${ }^{2}$ Michel Foucault, L'Usage des plaisirs, op. cit., p. 10.

${ }^{3}$ Michel Foucault, "Conversazione con Michel Foucault», dans Id., Dits et écrits, t. 2, op. cit., p. 862,886 .

${ }^{4}$ Voir par ex. Michel Foucault, « Préface à la transgression », dans Id., Dits et écrits, t. 1, op. cit., p. 261-278. 
viabilité : car l'expérience littéraire trouvait le lieu de sa possibilité au point de son effondrement, dans la mort ou la folie), en une ascèse du péril que résume la notion d'essai, caractéristiquement tendue entre esthétique et éthique.

Informé de ceci, on s'étonnera peut-être moins de constater que Musil retrouvait déjà le sens le plus fondamental de la notion d'essai, bref sa signification éthique, en la rapportant au « risque » 1 d'effondrement qu'un tel travail transformateur de soi sur soi comporte toujours. C'est bien en opérant un déplacement vers l'éthique d'une notion d'origine littéraire qu'il en viendra à écrire, dans L'Homme sans qualités, que, tout bien considéré, « un essai est la forme unique et inaltérable qu'une pensée décisive fait prendre à la vie intérieure d'un homme »; c'est selon lui précisément cette dimension éthique qu'occulterait le mot allemand de Versuch et dont rendrait mieux compte le vocable français d'« essai » ${ }^{2}$.

Ce qui précède autorise à prendre une vue plus large sur la question de la forme de la subjectivité en général telle qu'elle s'esquisse dans l'œuvre de Musil. L'idée essentielle est que ce sera l'absence originaire d'une telle forme qui doit permettre de rendre compte non seulement des formes diverses de captures dont le sujet peut devenir l'objet dans tel ou tel contexte socio-historique, mais encore de son aptitude à se transformer, par essais, « dans sa vie intérieure », afin d'approcher de quelque chose comme "l'autre état $»^{3}$.

${ }^{1}$ Robert Musil, « De l'essai », art. cit., p. 334.

2 Robert Musil, L'Homme sans qualités, op. cit., p. 305. Signalons que le mot Versuch est fréquent dans les textes de Kant ne relevant plus directement de la faculté de juger déterminante mais faisant droit - ainsi les textes historicopolitiques - à l'exercice d'une libre réflexion dans le jugement : il renvoie alors au risque de l'errance et de l'erreur qui s'empare de la pensée et de son sujet lorsqu'il «ose faire un pas sans la roulette d'enfant» (Emmanuel Kant, Vers la paix perpétuelle, Que signifie s'orienter dans la pensée, Qu'est-ce que les Lumières? et autres textes, trad. fr. J.-F. Poirier et F. Proust, Paris, Éditions Flammarion, coll. «GF Flammarion», 2006, p. 44). À la limite, ce concept de l'expérience comme essai indique ce qui, du côté de l'éthique, résiste chez Kant au concept gnoséologique d'Erfahrung, et par suite à son appropriation néo-kantienne, par exemple chez Hermann Cohen - ou chez le professeur de Törless.

${ }^{3}$ Entre ces deux pôles (celui d'une «capture» assujettissante ; celui d'un essai producteur d'une forme inédite du soi), il importe de faire droit à la pensée de Lukács. On sait que l'essai est conçu par celui-ci, en 1910, comme un travail de l'art producteur d'une « forme » en ce sens précis qu'il offre un lieu où se réconcilient les modalités déchues de «la vie » empirique et l'essentialité de "la vie » même (voir Georg Lukács, « À propos de l'essence et de la forme de l'essai », dans Id., L'Âme et

122 
$\mathrm{Au}$ « point de départ » du raisonnement musilien gît le « Théorème de l'amorphisme »: celui-ci condense en quelques phrases toute une vision de l'essence de l'homme, il offre presque les linéaments d'une anthropologie philosophique, entendu cependant qu'il n'y a, selon l'auteur, nulle essence de l'homme ni même du sujet à chercher derrière les formes que lui impose l'organisation sociale; c'est bien plutôt par et dans ces formes sociohistoriques seules qu'il découvrira les conditions (les limites) et les moyens de son devenir-sujet :

Si nous essayons d'abstraire de nous-mêmes ce qui n'est que convention inhérente à l'époque, il reste quelque chose de tout à fait amorphe [...]. L'homme n'existe que dans des formes qui lui sont fournies du dehors [...]. C'est l'organisation sociale qui donne à l'individu sa forme d'expression ${ }^{1}$.

La thèse de ce que l'on pourrait nommer une fabrication intégrale de l'homme à la faveur des formes socio-historiques où il s'insère est ici exprimée dans sa plus grande radicalité : elle entend en effet rendre compte jusqu'au contenu de la vie intérieure du sujet, jusqu'aux "sentiments euxmêmes »; c'est d'ailleurs pourquoi elle peut aussi permettre d'expliquer l'attachement extrême que le sujet portera toujours à ces formes, y compris dans leur caractère d' " extrême cruauté " (par exemple en régime capitaliste): c'est qu'il n'aurait, en leur absence, «ni consistance, ni possibilité d'expression $»^{2}$. En accord avec ce que nous explorions plus haut, le théorème de l'amorphisme renvoie à une double condition critique, épistémologique, d'une part, socio-politique, de l'autre. D'une façon, elle prend acte dans son orbe propre de la crise de la représentation (la crise du rapport du langage à son référent désormais problématique, le réel) qui n'autorise plus la position apriorique d'un sujet autocentré, souverain et constitutif d'une objectivité stable (crise décrite par Gargani et que tente de

les formes, trad. fr. G. Haarscher, Paris, Éditions Gallimard, coll. « Bibliothèque de Philosophie », 1974, p. 12-33; et le récapitulatif critique qu'offre de cette problématique Theodor W. Adorno, «L'Essai comme forme », dans Id., Notes sur la littérature, trad. fr. S. Muller, Paris, Éditions Flammarion, coll. «Champs», 2009, p. 5-29). Dans cette perspective, Histoire et conscience de classe correspond à l'approfondissement - par son étude au plan économique, social et idéologique du moment de la «capture " (la vie empirique devient réification) et à l'étude des conditions de son renversement possible (l'essence de la vie comme conscience de classe, entre discipline existentielle et organisation collective dans la forme du parti). ${ }^{1}$ Robert Musil, «L'Allemand comme symptôme », art. cit., p. 349.

${ }^{2}$ Ibid., p. 353. 
résoudre Husserl par les moyens de la phénoménologie). Mais surtout, et sans doute plus profondément, elle renvoie à la perte de repères au sein même de l'expérience subjective la plus intime qu'emporte avec elle la mécanisation bureaucratique du monde capitaliste au début $\mathrm{du} \mathrm{XX} \mathrm{X}^{\mathrm{e}}$ siècle (et que décrit Lukács dans le but de déterminer le sujet qui, en contradiction interne avec ce système, a pour mission historique d'en opérer la négation totale, soit le prolétariat). On l'a compris, chez Musil, le renversement positif de cet amorphisme principiel s'oriente vers une pratique de l'essai, une pratique pensée ( «dans les arts») et vécue (dans le « chaos des relations privées »), dont le fondement est une transformation du soi qui le conduirait à une expérience enrichie de la vie intime et sociale ; mais c'est aussi bien dire que cette expérience est, pour l'heure, confisquée par des formes de pensée et d'existence qui appauvrissent et réduisent cette expérience ; c'est enfin affirmer qu'une telle transformation de soi doit être intimement liée à une expérience de type cette fois politique.

Deux moments, donc, sur fond d'amorphisme : avec cette idée forte, Musil peut d'une part nous aider à comprendre ce que l'on nommerait, par exemple avec Benjamin, la pauvreté en expériences (Erfahrungen) typique $\mathrm{du}$ monde moderne ${ }^{1}$ et, en quelque sorte, la condition structurale du phénomène de la réification, de la dissolution de toute expérience vécue en

1 À cet égard, Benjamin oppose l'Erfahrung — l'expérience comme mouvement intégrateur à longue durée fondée sur la transmissibilité de la mémoire, et qui permet à l'individu de se hisser au rang de personne - et l'Erlebnis - l'expérience vécue partielle, séparée et éphémère absolument non-cumulative, favorisée par la division du travail et du monde spatio-temporel en segments discontinus —, par exemple à propos de Baudelaire : "Telle est l'expérience vécue que Baudelaire a prétendu élever au rang de véritable expérience : il a décrit le prix que l'homme moderne doit payer pour sa sensation : l'effondrement de l'aura dans l'expérience vécue du choc » (Walter Benjamin, Charles Baudelaire. Un poète lyrique à l'apogée du capitalisme, trad. fr. J. Lacoste, Paris, Éditions Payot, coll. « Petite Bibliothèque Payot», 1990, p. 207). Dans sa recherche personnelle, Benjamin, tournant le dos à l'Erlebnis, envisagera un retour à l'Erfahrung kantienne (voir Walter Benjamin, «Sur le programme de la philosophie qui vient », trad. fr. M. de Gandillac, dans Id., Euvres I, Paris, Éditions Gallimard, coll. « Folio », 2011, p. 179-197), mais enrichie de ce qui excède les limites de celle-ci et qu'il découvre, notamment, dans le surréalisme (voir Walter Benjamin, «Le surréalisme. Le dernier instantané de l'intelligentsia européenne », trad. fr. M. de Gandillac, dans Id., Euvres II, Paris, Éditions Gallimard, coll. « Folio », 2011, p. 113-134). S'il n'est pas impossible que cette recherche incite progressivement Benjamin à céder sur l'instance subjective (c'est en tout cas l'interprétation d'Adorno), il n'en demeurera pas moins attentif, notamment sous l'influence de Brecht, au problème de l'organisation.

\section{4}


première personne ; mais il nous invite aussi à questionner la nécessité de son renversement - y compris politique - en tout autre chose, par essais et tentatives, vers un «autre état» de soi et du monde. Quant au premier point, négatif, nous nous contenterons de citer un passage tout à fait remarquable de L'Homme sans qualités :

Jadis, l'on avait une meilleure conscience à être une personne qu'aujourd'hui. Les hommes étaient semblables à des épis dans un champ ; [...] ce qui restait à l'épi isolé de mouvements personnels était quelque chose de clairement défini dont on pouvait aisément prendre la responsabilité. De nos jours, au contraire, le centre de gravité de la responsabilité n'est plus en l'homme, mais dans les rapports des choses entre elles. N'a-t-on pas remarqué que les expériences vécues se sont détachées de l'homme ? [...] Il s'est constitué un monde de qualités sans homme, d'expériences vécues sans personne pour les vivre ; on en viendrait presque à penser que l'homme, dans le cas idéal, finira par non plus pouvoir disposer d'une expérience privée $[\ldots]$; la plupart des hommes commencent à tenir pour naïveté que l'essentiel, dans une expérience, soit de la faire soi-même, et dans un acte, d'en être l'acteur ${ }^{1}$.

Il serait légitime, et sans nul doute nécessaire, de replacer une telle description - qui renvoie, mutatis mutandis, à celle que Lukács propose de la réification - dans une topologie plus large des formes de la dépossession subjective correspondant aux développements historiques du mode de production capitaliste: ainsi étudierait-on, outre la réification taylorofordiste, l'aliénation au sens du jeune Marx, tout comme le « fétichisme de la marchandise » et la domination $\mathrm{du}$ 《 travail mort» sur le «travail vivant » qui sont au centre des œuvres de la maturité ; mais on prolongerait aussi l'analyse vers la " privatisation » (Castoriadis) de l'individu " unidimensionnel » (Marcuse) dans le cadre du capitalisme de compromis propre à l'aprèsguerre, avant d'aborder les formes "narcissiques » (Lasch) et tendancielle-

\footnotetext{
${ }^{1}$ Robert Musil, L'Homme sans qualités, op. cit., p. 178-179 (nous soulignons). Pour illustrer cet extrait, on peut encore penser, dans un contexte intellectuel proche, à un passage hilarant, mais glaçant, de Siegfried Kracauer, Les Employés. Aperçus de l'Allemagne nouvelle (1929), trad. fr. C. Orsoni, Paris, Éditions Les Belles lettres, coll. « La Couleur des Idées », p. 75 : «L'aberration qui fait surgir les principes de la correspondance commerciale dans un domaine où ils n'ont pas leur place resterait une bizarrerie si, dans les lettres elles-mêmes, les sentiments n'étaient pas eux aussi enfermés dans une camisole de force. Appelons la destinataire Käthe. Ils ne s'adressent pas l'un à l'autre par leurs prénoms, mais s'appellent "jeune collègue". "Cher jeune collègue", écrit la jeune fille de 19 ans [...]."Quelle est notre position concernant les rapports sexuels en général ? demande Käthe" ».
} 
ment schizophréniques caractéristiques quant à elles du capitalisme tardif ou «postmoderne » (F. Jameson). Dans tous les cas : pauvreté en expériences, amenuisement corrélatif de la sphère du rapport à soi, donc de la possibilité même d'une constitution de soi par soi comme personnalité ou individu autonome.

Mais une telle expérience subjective de la dépossession, précisément parce qu'elle est intégralement relative à une expérience socio-historique objective appauvrissante, et que tout en lui demeurant interne elle la contredit aussi partiellement, renvoie toujours en droit à la possibilité de son dépassement dialectique, en tant qu'un tel renversement subjectif suppose et implique lui-même la transformation — la dé-formalisation - de l'expérience elle-même dans son objectivité. Dans le cas de Musil, comme on l'a vu, il prend la forme éthico-esthétique de l'essai discipliné ; mais, logiquement, celui-ci n'est pas pour lui séparable d'une réflexion quant au mode d'organisation politique apte à assurer ledit renversement.

Conformément à la thèse de l'amorphisme a priori du sujet, on conçoit que l'approche de "l'autre état» ne puisse être entièrement du ressort d'une décision éthique, d'une réforme individuelle du caractère. La transformation subjective appelle en fait une modification des conditions mêmes de la formation du sujet - et réciproquement : on peut ici pointer une nécessaire circularité de la révolution intérieure et du bouleversement socio-historique. D'un mot, «l'événement radicalement nouveau» demeure insuffisant en l'absence de «la création des conditions sociales capables d'assurer aux efforts idéologiques une stabilité et un tirant d'eau suffisant ${ }^{1}: c^{1}$ 'est du reste dans une telle création - et c'est elle qui pose le problème, collectif, de l'organisation —, que réside selon Musil le véritable "héroïsme ». Pour autant, on sait que cette création restera sans effets, si même elle n'est pas vouée au pire, tant qu'elle n'aboutit pas, chez le sujet lui-même, par-delà les formes de sa réification (notamment ratioïdes), à une reconquête de sa personnalité, par une re-signification intentionnelle des formes sociales objectives, débouchant sur un nouvel union du sentiment et de la pensée ${ }^{2}$. C'est alors encore une fois le problème de la discipline réclamé par la

\footnotetext{
${ }^{1}$ Robert Musil, « L'Allemand comme symptôme », art. cit., p. 138, 152.

${ }^{2}$ Un tel rapport de réciprocité circulaire, sur fond d'a-morphisme subjectif radical, permet de comprendre que Walter, le meilleur ami d'Ulrich, puisse tour à tour s'accuser lui-même et l'époque de ses échecs : «Si c'était lui jusqu'alors qui se montrait incapable de travailler et se jugeait mauvais, c'était l'époque, maintenant, qui se révélait incapable, et lui qui se trouvait sain » (Robert Musil, L'Hommes sans qualités, t. 1, op. cit., p. 73).
} 
réforme éthique de soi qui se pose ; car, comme l'écrit encore Musil : «Rien n'est plus étranger à l'essai que l'irresponsabilité et l'inachèvement des inspirations qui relèvent de la subjectivité $»^{1}$.

\section{Une conclusion ouverte}

Nous choisissons en guise de conclusion de rapprocher deux longues citations. La première d'entre elles doit donner une idée de la manière dont la problématique de la discipline éthique pourra être reprise et comme radicalisée chez un auteur qui, quoique plus tardif, n'en appartient pas moins de plein droit à la constellation intellectuelle décrite ici. Dans un roman intitulé Extinction (1986), Bernhard écrit ceci :

C'est une attitude déplaisante que de croire que l'enrichissement de l'esprit n'est plus nécessaire, qu'un élargissement des connaissances, quelles qu'elles soient, est superflu, qu'une formation poursuivie du caractère est une perte de temps. En quittant le lycée, ils ont très tôt cessé d'élargir leurs connaissances et de former leur caractère, avant même leur vingtième année ils ont donc renoncé au travail sur soi et se sont contentés de leur acquis, avec une suffisance grossière [...]. Déjà vers la vingtième année ils avaient renoncé, je dois le dire, n'avaient plus rien laissé pénétrer en eux, ne s'étaient plus donné aucune peine, avaient reculé devant le moindre effort pour s'améliorer. Pourtant il va de soi qu'on élargisse ses connaissances, qu'on forme et fortifie son caractère tant qu'on est en vie. Car celui qui cesse d'élargir ses connaissances et de fortifier son caractère, c'est-à-dire de travailler sur soi afin de tirer de soi le meilleur parti possible, a cessé de vivre [...]. Ils sortent de l'école et restent bloqués et ne font plus aucun effort. Et s'effondrent, comme on peut bien le dire. Et l'homme qui ne fait pas d'effort est sans aucun doute un homme répugnant, nous ne pouvons le regarder, si nous le regardons, sans la plus grande répulsion. Il nous déprime, non seulement nous rend malheureux à la longue, mais furieux. Nous nous opposons à lui, mais cela ne sert à rien. L'ensemble des gens ne se donnent du mal, dirait-on, qu'aussi longtemps qu'ils peuvent attendre des diplômes stupides avec lesquels ils peuvent se pavaner en public, lorsqu'ils ont en main un nombre suffisant de ces diplômes stupides, ils se laissent aller. La plupart ne vivent que pour obtenir des diplômes et des titres, pour nulle autre raison, et quand ils ont obtenu à leur avis un nombre suffisant de diplômes et de titres, ils se laissent choir dans le lit douillet de ces diplômes et de ces titres. Ils n'ont, dirait-on, aucun autre but dans la vie. Une vie personnelle, indépendante, une existence personnelle, indépendante, cela ne les intéresse pas du tout, dirait-

${ }^{1}$ Ibid., p. 305. 
on, ils ne s'intéressent qu'à ces diplômes et à ces titres sous lesquels le genre humain risque d'étouffer depuis des siècles ${ }^{1}$.

Nous rapprocherons cet extrait d'un autre, tiré d'un livre classique, dont Musil était grand lecteur. Voici le portrait fiévreux de l'humaniste tel que le peint Burckhardt dans un célèbre passage de La Civilisation de la Renaissance en Italie (1860) :

Et c'est ainsi qu'ils se lançaient dans une vie agitée, dévorante; tout à tour précepteur, secrétaire, professeur, valet des princes, se consumant dans des études ingrates, en butte à des inimitiés mortelles, à des dangers incessants, élevé aux nues ou accablé de mépris, opulent aujourd'hui, demain misérable, il est l'image vivante de l'instabilité [...]. Mais le grand mal était que cette condition ne comportait guère une demeure fixe, attendu qu'elle rendait les changements de résidence nécessaires ou qu'elle empêchait l'individu de se plaire longtemps quelque part. Se fatiguant lui-même de ceux au milieu desquels il vivait, se sentant mal à l'aise parmi des ennemis acharnés à le perdre, il finissait par se décourager et par lasser un entourage amoureux de la nouveauté. Malgré tout ce qui, dans cette situation, rappelle les sophistes du temps des empereurs, tels que les décrit Philostrate, ceux-ci étaient dans une condition meilleure, ils étaient généralement riches ou se résignaient plus aisément aux privations ; en somme, leur vie était facile [...]. L'humaniste de la Renaissance, au contraire, est obligé de posséder une vaste érudition et de savoir se plier aux situations et aux occupations les plus diverses. Pour s'étourdir, il use du plaisir et en abuse ; on le croit capable de tout, et, en effet, il se met au-dessus de toutes les lois de la morale vulgaire. On ne saurait concevoir de pareils caractères sans un orgueil qui résiste à tout; ils en ont besoin, ne fût-ce que pour rester supérieurs aux événements ; la haine et le culte dont ils sont tour à tour l'objet fortifient nécessairement en eux ce sentiment. Ils sont les exemples les plus remarquables de la subjectivité livrée à elle-même ${ }^{2}$.

La pertinence de ce rapprochement s'éclairera peut-être si l'on s'avise que l'on a ici affaire à deux moments situés aux extrémités d'un spectre temporel : celui du travail socio-historique de mise en forme d'une subjectivité de prime abord a-morphique, vide. Retenons que « l'individu » est avant

1 Thomas Bernhard, Extinction. Un effondrement, trad. fr. G. Lambrichs, Paris, Éditions Gallimard, coll. «Folio », 1999, p. 75-77. L'exergue du livre est tiré des Essais de Montaigne, un des principaux essayistes aux sens de Lukács.

${ }^{2}$ Jacob Burckhardt, La Civilisation de la Renaissance en Italie, trad. fr. H. Schmitt, Paris, Éditions Bartillat, 2012, p. 316. 
toute chose «la forme que reflète le processus social ${ }^{1}:$ le caractère si remarquable de l'humaniste correspond alors à la prise de conscience de l'individualité relative à l'émergence de la Cité moderne - mais à quoi renvoie dans ce cas l'effort solitaire et semble-t-il désespéré de Bernhard ? Tout simplement à ce qui demeure, chez certains individus exceptionnels, d'une aptitude à l'individualisation esthétique, à la construction autonome d'un soi, en l'absence radicale des conditions politiques qui permettraient à cet effort de trouver son sens et sa destination. On a vu comment Musil pouvait pour sa part tenter de tenir ensemble - fût-de façon fragmentaire éthique, esthétique et politique dans une pratique pensée de l'essai censée approcher de «l'autre état »; mais s'il peut tenir tout cela ensemble, n'est-ce pas parce que, écrivant soixante ans avant Bernhard, il pouvait encore sentir à l'œuvre les forces historiques collectives aptes à relayer une forme d'approfondissement existentiel qui, au mieux, trouvait sa seule finalité dans une expérience enrichie de soi et du monde individuelle? À ce titre, ce rapprochement est fondé et significatif, au moins à une époque qui a montré de tant de façons, souvent attristantes, que l'« état dans lequel l'individu disparaît » peut aussi bien être «celui de l'individualisme forcené »; peutêtre peut-il en effet permettre de nous souvenir que, dans un pareil contexte, il est inutile de "sacrifier l'individu lui-même ", mais qu'il est en revanche on ne peut plus urgent «de faire porter [la] critique sur le principe de l'individuation de la société lui-même $»^{2}$. Telle est selon nous l'unique manière d'enfin donner un contenu politique consistant au thème, sinon consternant, de l'esthétique de l'existence ${ }^{3}$.

${ }^{1}$ Theodor W. Adorno, Minima Moralia. Réflexions sur la vie mutilée, trad. fr. É. Kaufholz et J. R. Ladmiral, Paris, Éditions Payot, "Petite Bibliothèque Payot», 2005, § 147, p. 307.

${ }^{2}$ Ibid., § 97, p. 201-202.

${ }^{3}$ Peut-être cette ultime réflexion permettrait-elle enfin de comprendre une des raisons (inconscientes) d'une certaine tentation de l'Italie commune à plusieurs auteurs autrichiens après 1945. Si l'Italie a alors pu apparaître comme un cadre correct à partir duquel s'essayer à une (re-)construction de soi par soi, à rebours de la haine de soi qui semble marquer, surtout depuis l'après-guerre, notamment en arts plastiques, le destin intellectuel de l'Autriche, n'est-ce pas parce que ce pays fut le dernier en Occident (lors de ce qu'il est convenu d'appeler la séquence rouge italienne) où a été posé explicitement, et intensément, le problème d'une organisation politique (située par delà les formes du Parti et de l'État) capable de prendre en charge une transformation radicale de l'existence individuelle et collective, ceci dans le contexte d'un capitalisme imposant (depuis l'accroissement délirant de la sphère de la consommation, notamment culturelle) des formes tout à fait neuves d'appau-

\section{9}


vrissement de l'expérience vécue ? Une telle tentation de l'Italie est en tout cas le thème général du livre de Bernhard évoqué ici ; c'est enfin le motif déterminant de l'existence d'une autre grande écrivaine autrichienne, native, comme Musil, de la ville de Klagenfurt, Ingeborg Bachmann — c'est bien à Rome que celle-ci s'est éteinte. 